\title{
A nonoverlapping heterogeneous domain decomposition method for three-dimensional gravity wave impact problems
}

\author{
Yi Zhang ${ }^{\mathrm{a}}$, Solomon C. Yim ${ }^{\mathrm{a}, *}$, Facundo Del Pin ${ }^{\mathrm{b}}$ \\ ${ }^{a}$ School of Civil $\&$ Construction Engineering, Oregon State University, Corvallis, OR 97331, USA \\ ${ }^{b}$ Livermore Software Technology Corporation, Livermore, CA 94551, USA
}

\begin{abstract}
A 3D heterogeneous flow model which combines incompressible viscous flow and potential flow both with freesurface boundaries is developed and solved numerically, using finite element method and boundary element method, respectively. The coupled model is solved based on a nonoverlapping domain decomposition method, which reduces the problem to the coupling interface. At the interface, the problem is equivalent to Bernoulli's equation consisting of mappings from potential/velocity to velocity/pressure. These mappings enable us to design the scheme following the Dirichlet-Neumann method for nonoverlapping domain decomposition, and lead to a staggered scheme. We also discuss data transfer as well as free surface reconstruction at the interface. Errors introduced by the surface reconstruction technique are examined and discussed in detail. The errors from the first-order staggered scheme is also studied. A comparison of numerical prediction with experimental data that the proposed method performs efficiently and accurately for wave impact problems.
\end{abstract}

Keywords: heterogeneous domain decomposition, boundary element method, finite element method, wave impact, potential flow, viscous flow, Navier-Stokes equations

\section{Introduction}

Gravity wave impact problems have been the subject of investigation in the past several decades, due to their challenging highly nonlinear nature and importance in offshore engineering [1], naval architecture [2] and coastal engineering [3]. Because of the transient nature of the problem and the arbitrary, complex geometry of the structures in industrial applications, a time domain study in physical space is required. Accompanied by advances in numerical methods, techniques in modeling free-surface flows in the time domain, such as the one pioneered by Longuet-Higgins and Cokelet [4] in tracking free surface using the mixed-Eulerian-Lagrangian method (MEL), have been gaining a steady interest. An integrated simulation environment for wave-structure interaction, often referred to as a numerical wave tank (NWT), assembles techniques of modeling wave generation, propagation, dissipation and interaction with structures. The past three decades have witnessed extensive development and studies on this topic. The modeling technique introduced in this paper is part of an on-going effort in the development of a robust and practical NWT.

Regarding the modeling of flows in a NWT, two approaches exist for the time domain wave simulation. The first and also the most popular one, is to use the homogeneous flow, by which a single model is used to describe the entire flow domain. The model could be viscous flow, governed by the Navier-Stokes equations (NSE), such as the work by Lin and Liu [5], Park et al. [6], Yuan et al. [7], and Li et al. [8]. It could also be a simplified model such as potential flow (PF) or Boussinesq model, such as the work by Beji and Battjes [9], Grilli et al. [10], Ryu et al. [11], Liu et al. [12] and Nimmala et al. [13]. An extensive review of using PF solutions to NWT using the boundary integral equation (BIE) approach is given by Kim et al. [14].

\footnotetext{
${ }^{*}$ Corresponding author

Email addresses: zhangy2@onid.oregonstate.edu (Yi Zhang), solomon.yim@oregonstate.edu (Solomon C. Yim), fdelpin@lstc.com (Facundo Del Pin)
} 
In the second approach, the flow domain is decomposed into multiple subdomains, and in each subdomain a different flow model is adopted. This is sometimes referred to as a heterogeneous flow model [15, chap. 8]. The motivation of this approach is of two fold. First, the development of a large scale solver like NWT is often based on legacy codes, frequently originated from other purposes. For example, the wave generation/dissipation and viscous flow solution techniques have separate origins in coastal engineering and aerospace engineering, respectively. It is pointed out recently that the approach of combining code bases from different fields is the most common path taken by multiphysics solver developers, because it reduces development overhead and takes advantage of previous validations [16]. In the context of a NWT, such a multiphysics model often contains a NSE solver coupled with simplified models, such as a PF or a Boussinesq model, with the NSE describing the "near field" such as flow near a body or in the shoaling zone, and the simplified models describing the "far field". The second motivation of a heterogeneous flow model is to reduce computing cost. It is well known that though a NSE numerical solver captures viscous and vortex effects that can not be computed in simplified models without empirical modeling parameters (see e.g., [17]), its computing cost is rather high, due to the nonlinearity and the propensity for instability induced by the incompressibility condition. On the other hand, for far field wave propagation, simplified models are sufficient and more efficient, as validated by numerous works in the past century (see, e.g., [18, 19]).

Because of the spatial decomposition the flow domain, the modeling problem can be cast into the framework of (heterogeneous) domain decomposition (DD) methods. Early works on numerical applications of the DD methods include Bjørstad and Widlund [20], Cambier et al. [21], Dinh et al. [22], Dryja [23] and Glowinski et al. [24], among others. Recent overviews on this topic include the reviews by Xu and Zou [25], $\mathrm{Xu}$ [26], the books by Mathew [27], Quarteroni and Valli [15], Toselli [28] and Wohlmuth [29]. In particular, the well-posedness of the problems of NSE coupled with Stokes flow or Oseen flow have been studied by Fatone et al.. [30, 31], Feistauer and Schwab [32, 33], and Schenk and Hebeker [34].

Examples of the free surface flow numerical solvers based on the DD approach include the work by Campana et al. [35], Iafrati and Campana [36], Colicchio et al. [37], Sitanggang and Lynett [38], Kim et al. [39], Hamilton and Yeung [40], and Zhang et al. [41]. Among these works, all except [40] are 2D models. In [40], a non-overlapping coupled 3D solver based on the scheme proposed by Iafrati and Campana [36] is reported. In their study, the solver adopts the method of Green's function for the far field and a pseudo-spectral method for the viscous subdomain. Since the Green's function used is specifically developed for an axial symmetric domain as in the problem of the interaction between waves and a cylindrical structure, it is very efficient but also limited in application. Moreover, the solver is limited to a linearized free-surface boundary, and no wave generation is incorporated in the far field model.

In this paper we present the progress made toward a 3D DD solver, based on our 2D development and theoretical study, reported in Zhang et al. [41] and Zhang et al. [42], respectively. In particular, the relationship between the coupling scheme and the Dirichlet-Neumann (D-N) method for a homogeneous elliptic problem [20,43] is discussed in Zhang et al. [41]. The scheme is related to the solution for the Schur's complement formulation of the coupled problem, as discussed in Zhang et al. [42]. In this paper, we follow this D-N-like approach, to study a 3D potential flow-viscous flow heterogeneous model, with nonlinear free surface tracking/capturing capability. The PF subdomain is solved using a boundary element method (BEM), whereas NSE subdomain is solved using a finite element method (FEM). The BEM solver is based on the work of Grilli et al. [10, 44], Fochesato and Dias [45], and Nimmala et al. [13]. The BIE from the Laplace equation is discretized using a collocation BEM, and the linear system is solved using the generalized minimal residual method (GMRES) accelerated by the fast multipole method (FMM). On the other hand, the FEM solver for the NSE subdomain is based on a monolithic formulation and a pressure segregation scheme, proposed by Soto et al. [46-48] and Codina [49-51]. In this scheme, P1-P1 tetrahedron elements are used to discretize the domain. This type of element does not meet the Ladyzhenskaya-Babuška-Brezzi (LBB) condition [52-54], and the scheme provides stabilization toward pressure.

In the current study, we first further develop a 3D algorithm and implement it to the 3D NWT model. This includes introducing techniques for the interpolation and free surface matching at the interface (Sec. 3.2 and 3.3). Moreover, a numerical analysis for the error due to free-surface matching is performed on solitary wave propagation (Sec. 3.3). To further improve the mathematical robustness of our method, in the current study we further interpret the DD method using the Helmholtz decomposition, and explore the nature of the 
matching conditions as well as the corresponding operators (Sec. 4). A comparison of computing time is also shown to demonstrate the efficiency of the proposed method which was not examined in the previous $2 \mathrm{D}$ studies.

The rest of this paper is organized as follows. In $\S 2$ we describe the coupled flow model. In $\S 3$, we introduce the DD scheme based on two mappings, corresponding to the PF and NSE solution, respectively. We also discuss numerical techniques for data transfer and free surface reconstruction near the interface. In $\S 4$, we explore the mathematical foundation of the coupling scheme using the Helmholtz decomposition. In $\S 5$, two numerical examples are reported to validate the 3D DD model. In particular, we discuss spatial and temporal convergence of the method, as well as its efficiency in terms of computing time. Further discussions and conclusions are presented in $\S 6$.

\section{Heterogeneous flow model}

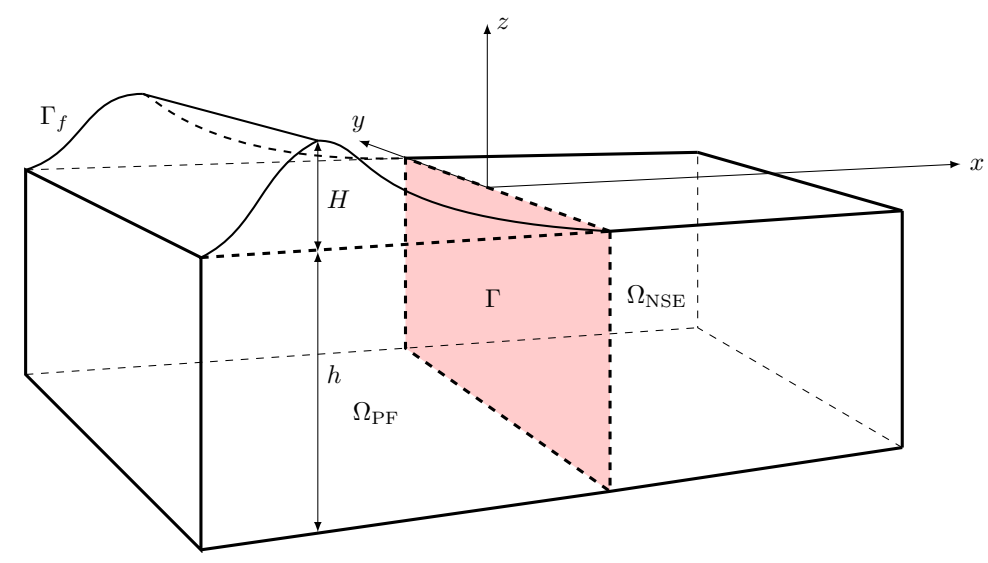

Figure 1: Non-overlapping decomposition of a wave tank flow domain. $\Omega_{\mathrm{NSE}}$ and $\Omega_{\mathrm{PF}}$ indicate viscous flow and potential flow subdomain, respectively. $h$ is the water depth and $H$ the wave height.

In our DD model, as shown in Figure 1, the flow domain $\Omega \subset \mathbb{R}^{3}$ is decomposed into two non-overlapping subdomains $\Omega_{\mathrm{NSE}}$ and $\Omega_{\mathrm{PF}}$, within which incompressible viscous flow and potential flow models are adopted, respectively. $\Gamma=\partial \Omega_{\mathrm{NSE}} \cap \partial \Omega_{\mathrm{PF}}$ is the interface between the subdomains. Though only the case of twosubdomain decomposition is presented in this paper, such philosophy applies to cases with a more complex decomposition. In wave-structure interaction applications, $\Omega_{\mathrm{PF}}$ denotes the "far field" and $\Omega_{\mathrm{NSE}}$ the "near field" of the domain, and the latter interacts with the structure domain(s). In this paper we focus on the initial impact of the waves, and the numerical tests show that it suffices to model the structures as rigid. Though $\Gamma$ could be a general surface (2D manifold), in our implementation a vertical surface in the Eulerian representation is used for simplicity. 
The governing equations for our heterogeneous model are

$$
\begin{aligned}
\rho \frac{D \boldsymbol{u}}{D t}=-\nabla p+\mu \nabla^{2} \boldsymbol{u}+\boldsymbol{f}, & \forall(\boldsymbol{x}, t) \in \Omega_{\mathrm{NSE}} \times[0, T], \\
\nabla \cdot \boldsymbol{u}=0, & \forall(\boldsymbol{x}, t) \in \Omega_{\mathrm{NSE}} \times[0, T], \\
\boldsymbol{u}=\boldsymbol{u}^{*}, & \forall(\boldsymbol{x}, t) \in\left(\partial \Omega_{\mathrm{NSE}} \cap \partial \Omega \backslash \Gamma_{f}\right) \times[0, T], \\
\nabla^{2} \varphi=0, & \forall(\boldsymbol{x}, t) \in \Omega_{\mathrm{PF}} \times[0, T], \\
\frac{\partial \varphi}{\partial n}=q^{*}, & \forall(\boldsymbol{x}, t) \in\left(\partial \Omega_{\mathrm{PF}} \cap \partial \Omega \backslash \Gamma_{f}\right) \times[0, T], \\
\frac{D \boldsymbol{x}}{D t}=\boldsymbol{u}, & \forall(\boldsymbol{x}, t) \in \Gamma_{f} \times[0, T], \\
\frac{D \varphi}{D t}=-g z+\frac{1}{2}|\nabla \varphi|^{2}-\frac{p^{*}}{\rho} & \forall(\boldsymbol{x}, t) \in \Gamma_{f} \times[0, T], \\
\nabla \varphi=\boldsymbol{u}_{\mathrm{NSE}}, & \forall(\boldsymbol{x}, t) \in \Gamma \times[0, T], \\
\frac{\partial \varphi}{\partial t}+\frac{1}{2}|\nabla \varphi|^{2}+g z=-\frac{p_{\mathrm{NSE}}}{\rho}, & \forall(\boldsymbol{x}, t) \in \Gamma \times[0, T],
\end{aligned}
$$

where the unknown variables $(\boldsymbol{u}, p, \varphi)$ are functions of spatial and temporal coordinates $(\boldsymbol{x}, t)$, with $\boldsymbol{u}$ being velocity, $p$ pressure and $\varphi$ velocity potential. Fluid property includes density $\rho$ and viscosity $\mu$. Boldface symbols and superscript " $*$ " indicate vectors and boundary data, respectively. For a field quantity $a, a_{i}=\left.a\right|_{i}$, $i=$ "NSE","PF", and $a_{\Gamma}=\left.a\right|_{\Gamma}$ denote the corresponding restriction of $a$. Material derivatives are denoted by $D \cdot / D t$. In Eq.(1), (1a)-(1b) are the NSE, while Eq.(1d) is the governing equation for potential flow in $\Omega_{\mathrm{PF}}$. Velocity and flux boundary conditions are described in Eq.(1c) and (1e), respectively. In our implementation, either nonslip or free-slip boundary conditions can be imposed on the walls and bottoms (see $\S 5$ for details). Wave is generated with a moving boundary (left wall in Figure 1), as a model for a piston motion wave maker in actual wave laboratories [55, 56]. At other walls and bottom of $\Omega_{\mathrm{PF}}$ we have $q^{*}=0$. Eq. (1f) $-(1 \mathrm{~g})$ are the kinematic and dynamic frees surface boundary conditions, with $g$ being the gravitational acceleration and and $p^{*}$ the atmospheric pressure (gauge pressure). Eq. (1h)-(1i) describe the matching conditions on $\Gamma$. For their derivation and physical interpretation, see [30, 32-34, 41].

\section{Coupling scheme based on domain decomposition method}

The key in our method is to compute the interface data, with which problem (1) becomes decoupled and the NSE and PF subproblem can be solved using FEM and BEM, respectively, as discussed in Appendix A and B. Here we briefly introduce the coupling scheme. Further details can be found in our previous studies on the 2D problems $[41,42]$. In the next section we will present a new interpretation of the coupling method using the Helmholtz decomposition.

The essence of our method is to reduce the problem to the interface, as in many DD methods (see [57] for a review for its application on elliptic problems), and is closely related to the substructure methods [58]. First we introduce two operators.

Definition 3.1. Given $\varphi_{\Gamma} \in \mathcal{C}^{1}(\Gamma)$, define $\left.\mathcal{M}\left(\varphi_{\Gamma}\right) \triangleq \nabla \xi\right|_{\Gamma}$ such that $\xi$ solves Eq. (1d), (1e), (1f) and (1g) with $\left.\xi\right|_{\Gamma}=\varphi_{\Gamma}$.

Definition 3.2. For $\boldsymbol{u}_{\Gamma} \in C^{1}(\Gamma)$ define $\left.p\right|_{\Gamma} \triangleq \mathcal{N}\left(\boldsymbol{u}_{\Gamma}\right)$ such that (v,p) solves Eq. (1a), (1b) on $\Omega_{1}$, with Eq.(1c), (1f) and (1g), with $\left.\boldsymbol{v}\right|_{\Gamma}=\boldsymbol{u}_{\Gamma}$.

With matching conditions (1h)-(1i), we propose a coupling scheme in the format of the Dirichlet-Neumann method $[20,43]$. At $\Gamma$ the velocity $\mathcal{M}(\varphi)$ is used as the boundary condition for the NSE in $\Omega_{\mathrm{NSE}}$, whereas the pressure $\mathcal{N}(p)$ is used to update the potential through Bernoulli's equation. When both the velocity and pressure are available at the interface, for unknown $\varphi=\varphi_{\Gamma}$ we have an ODE on $\Gamma$ :

$$
\frac{\partial \varphi}{\partial t}=-\frac{1}{2}|\mathcal{M}(\varphi)|^{2}-\mathcal{N}(\mathcal{M}(\varphi))-g z .
$$


Eq. (2) is the Bernoulli's equation on $\Gamma$, with $\boldsymbol{u}$ and $p$ represented by operators $\mathcal{M}$ and $\mathcal{N}$. Note that Eq. (2) contains not only the matching condition (1i), but also (1h), because $\mathcal{N}(\mathcal{M}(\varphi)$ ) implies using $\nabla \varphi$ as boundary data for the NSE on $\Omega_{\mathrm{NSE}}$. As a result, the coupled problem (1) on $\Omega$ is reduced to the interface. The solution of the PF and NSE sub-problems, are incorporated in the operator $\mathcal{M}$ and $\mathcal{N}$, respectively. These mappings were first introduced in the development of $2 \mathrm{D}$ coupled models [41, 42], and here they are extended naturally to 3D implementation.

Numerous suitable time integral schemes can be applied to solve for $\varphi$ in Eq. (2). In this paper, we use the forward Euler scheme. Extension to other integration schemes is natural, see [41, 42].

We solve for $\varphi^{n+1}$ from $\varphi^{n}$ from

$$
\frac{\varphi^{n+1}-\varphi^{n}}{\Delta t}=-\frac{1}{2}\left|\mathcal{M}\left(\varphi^{n}\right)\right|^{2}-\mathcal{N}\left[\mathcal{M}\left(\varphi^{n}\right)\right] / \rho-g z
$$

This is equivalent to the following staggered scheme. From time step $n$ to $n+1$, with $\varphi_{\Gamma}^{n}$, we perform the following:

1. Solve the PF problem in $\Omega_{\mathrm{PF}}$ to obtain the flow velocity at $\Gamma$ as $\boldsymbol{u}_{\Gamma}^{n}=\nabla \varphi^{n}$. This yields $\mathcal{M}(\varphi)$ in Eq.(7).

2. Solve the NSE problem in $\Omega_{\mathrm{NSE}}$ to obtain $\left.p^{n}\right|_{\Gamma}$. This yields $\mathcal{N}\left[\mathcal{M}\left(\varphi^{n}\right)\right]$ in Eq. $(7)$.

3. Calculate $\varphi^{n+1}$ using Eq. (3) and $\left.\left(\boldsymbol{u}^{n}, p^{n}\right)\right|_{\Gamma}$.

In the context of BEM, the format of $\mathcal{M}$ can be derived as follows. The details of the matrices and vectors can be found in Appendix B. To simplify the presentation, here we assume $\Gamma$ is the only Dirichlet boundary of $\partial \Omega_{\mathrm{PF}}$, and the rest of $\partial \Omega_{\mathrm{PF}}$ is Neumann boundary. It is easy to obtain the tangential component of $\mathcal{M}\left(\varphi^{*}\right)$ by calculate tangential derivative of $\varphi^{*}$ on $\Gamma$. Obtaining the normal component is equivalent to applying the Poincaré-Steklov operator. In BEM formulation (Appendix B.3), this means seeking $\Psi$ (the vector for the flux). The elimination of the unknown $\Phi$ (the vector for the potential) on $\partial \Omega_{\mathrm{PF}} \backslash \Gamma$ gives

$$
\left(K^{D}\left(K^{N}\right)^{-1} J^{N}-J^{D}\right) \Psi=\left(-K^{D}\left(K^{N}\right)^{-1} K^{N^{*}}+K^{D^{*}}\right) \Phi^{*}+\left(-K^{D}\left(K^{N}\right)^{-1} J^{N^{*}}+J^{D^{*}}\right) \Psi^{*} .
$$

Here $J$ and $K$ denote matrices from the single and double layer potential, respectively. The superscript $N$ and $D$ denote the corresponding Neumann and Dirichlet boundary condition, respectively. The "** indicates the imposed boundary data. Thus algebraically the normal component of $\psi=\mathcal{M}\left(\varphi^{*}\right)$ is obtained by solving the linear system (4), given interface data $\Phi^{*}$. Note that all the matrices in Eq. (4) are densely populated, due the ubiquitous Laplacian. In our implementation the linear problem is solved using GMRES with Jacobi preconditioner, accelerated by fast multipole method (FMM), see [45] for details.

Similarly, we can derive the algebraic representation of operator $\mathcal{N}$. Using the same notations as in Appendix A, we seek $\left.P\right|_{\Gamma}$ in the discretized NSE

$$
\left[\begin{array}{ccc}
F & B_{1} & B_{\Gamma} \\
D_{1 \Gamma} & -C_{11} & -C_{1 \Gamma} \\
D_{\Gamma 1} & -C_{\Gamma 1} & -C_{\Gamma \Gamma}
\end{array}\right]\left[\begin{array}{l}
U \\
P_{1} \\
P_{\Gamma}
\end{array}\right]=\left[\begin{array}{l}
f \\
0 \\
0
\end{array}\right]
$$

Here the pressure unknowns are divided into interior $\left(P_{1}\right)$ and interface $\left(P_{\Gamma}\right)$ ones. The other matrices in Eq. (Appendix A.3) are in the block form accordingly. Eliminating the interior (and other boundary) unknowns $\left(U, P_{1}\right)$, it is easy to see that applying operator $\mathcal{N}$ is equivalent to solve the linear system

$$
\left(\left[\begin{array}{ll}
D_{\Gamma 1} & -C_{\Gamma 1}
\end{array}\right]\left[\begin{array}{cc}
F & B_{1} \\
D_{1 \Gamma} & -C_{11}
\end{array}\right]^{-1}\left[\begin{array}{c}
B_{\Gamma} \\
C_{1 \Gamma}
\end{array}\right]+C_{\Gamma \Gamma}\right) P_{\Gamma}=\left[\begin{array}{ll}
D_{\Gamma 1} & -C_{\Gamma 1}
\end{array}\right]\left[\begin{array}{cc}
F & B_{1} \\
D_{1 \Gamma} & -C_{11}
\end{array}\right]^{-1}\left[\begin{array}{l}
f \\
0
\end{array}\right]
$$

for $P_{\Gamma}$. The matrix inverse in Eq. (6) is equivalent to solve the interior NSE. Recall the pressure Poisson equation (PPE) in the NSE (see, e.g., $[60,61]$ ), we see that system (6) is part of the discretized PPE, and the inverse of operator $\mathcal{N}$ is equivalent to the interface component of the PPE. 


\subsection{Computational complexity}

Since one of the motivations of the DD method is to reduce computing cost, let us estimate the effect of replacing FEM with BEM for a selected part of the domain. For a 3D FEM domain with $N$ nodes, the number of nodes in a BEM discretization for the same domain is of $O\left(N^{2 / 3}\right)$. A preconditioned linear solver, such as PCG, for a sparse system is approximately of linear complexity $O(N)$. On the other hand, BEM produces a linear system with densely populated matrix, which can be solved with linear complexity using the fast multipole method (FMM) [45]. Therefore, using BEM instead of FEM reduces the computation from $O(N)$ to $O\left(N^{2 / 3}\right)$.

Let us point out that in the above estimation the reduced number of variables from FEM (velocity, pressure and level-set function) to BEM (potential) is not considered, neither is the cost of (Newton-like or Picard-like) iterations of solving NSE.

\subsection{Interpolation at the interface}

To impose matching condition (1h)-(1i) at the interface, we need to first transfer data (pressure and velocity) through the nonconforming mesh, as shown in Figure 2 . Since $\Omega_{\mathrm{NSE}}$ is discretized using tetrahedron

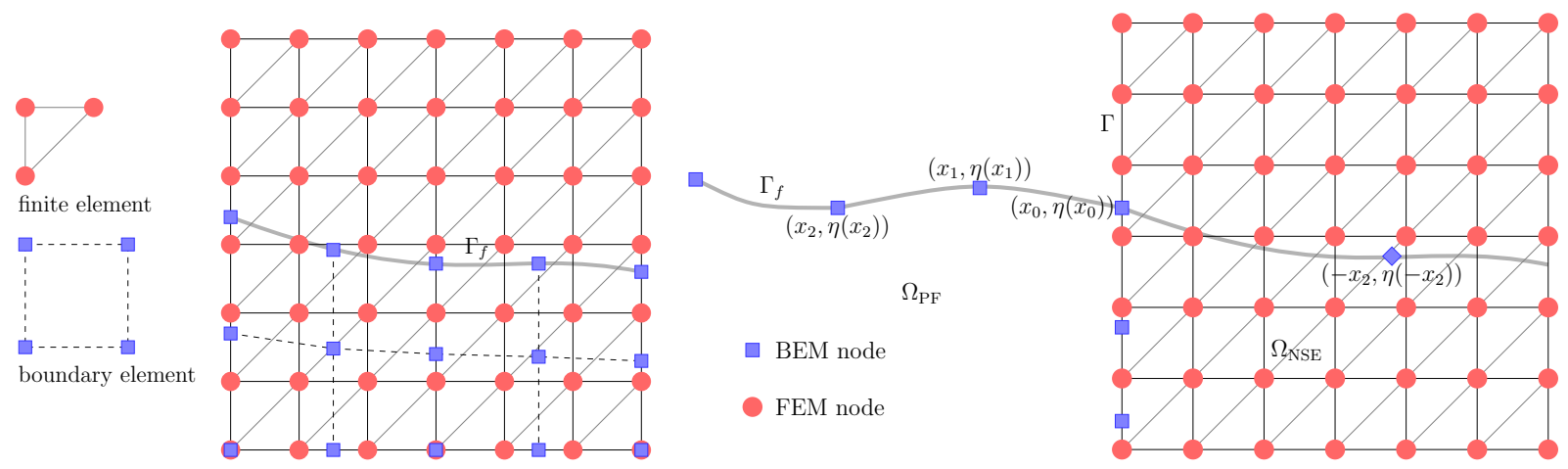

Figure 2: Nonconforming mesh at the interface. The $y-z$ plane view (left) shows that data are transferred through triangle (FEM) and quadrilateral (BEM) elements. The $x-z$ plane view (right) shows that free surface needs to be reconstructed using the Lagrangian nodes (BEM) and Eulerian data (FEM).

elements, on $\Gamma$ we have an unstructured mesh consisting of triangle elements. On the other hand, $\partial \Omega_{\mathrm{PF}}$ is discretized using a structured mesh of rectangular elements. Moreover, the mesh size in $\Omega_{\mathrm{NSE}}$ is in general much less than that of $\partial \Omega_{\mathrm{PF}}$. This is because the finite element mesh is refined to capture nonlinear free-surface effects such as jet and splash, as well as viscous and vortices effects.

To transfer data we use the same interpolation space of each subdomain. For a BEM node $\boldsymbol{x} \in \mathcal{T}_{e}$, with $\mathcal{T}_{e}$ being a P1-P1 triangle element, we have

$$
p(\boldsymbol{x})=\sum_{i=1}^{3} p^{i} \mathcal{I}_{i}(\boldsymbol{x})
$$

with $p^{i}$ being the nodal values at the vertices of $\mathcal{T}_{e}$, and $\mathcal{I}_{i}$ the shape function corresponding to vertex $i$ (usually in natural coordinates). Similar interpolation for FEM nodes is performed using the 4-node quadrilateral shape function from BEM.

\subsection{Matching of free surface}

The nodal elevation $\eta$ of the free surface $\Gamma_{f}$ from BEM and FEM should be matched, i.e., $\eta_{\mathrm{PF}}=\eta_{\mathrm{NSE}}=$ $\eta(x=0)$. The FEM and BEM solvers adopt different free surface capturing/tracking techniques. In $\Omega_{\mathrm{NSE}}$, a hybrid level-set method by Enright et al. [62] is used, while the mixed Lagrangian-Eulerian (MEL) method is adopted for $\Omega_{\mathrm{PF}}$. Due to the nature of the staggered scheme, we have to decide to which subdomain the updated free surface elevation should be imposed. If we impose a value on $\eta_{\text {NSE }}$ near $\Gamma$, in the level set 
setting this is equivalent to update all the nodal level set function values, as well as supplemental tracking particles. This is not only computationally inefficient but also may introduce a large gradient of the level set function. Therefore, we choose to impose the free surface elevation to $\eta_{\mathrm{PF}}$. Note that this does not change the coupling algorithm described below Eq.(7), as the free surface position is modified at end of each time step. To preserve a high order free surface profile near $\Gamma$, a free surface reconstruction is performed at every time step, as described in the following.

To reconstruct the free surface near $\Gamma$, we extend the interpolation technique in the $2 \mathrm{D}$ version of the $\mathrm{DD}$ method [41]. Let $M_{y}$ be the number of boundary elements in the $y$-direction. Because of the structured boundary mesh on $\partial \Omega_{\mathrm{PF}}$, the set of all the BEM nodes $S_{B}$ consists of $M_{y}+1$ mutually exclusive sets

$$
S_{B}^{i}=\left\{\boldsymbol{x} \mid \boldsymbol{x} \in S_{B} ; y=y^{i}\right\}, \quad i=1, \ldots, M_{y}+1 .
$$

Each $S_{B}^{i}$ corresponds to BEM nodes on a plane $y=$ constant, as a 2D slice of the 3D flow domain (Figure 2). On each $S_{B}^{i}$, we use an interpolation to reconstruct the free surface near $\Gamma$. Specifically, let $x_{1}$ and $x_{2}$ be the $x$-coordinate of the two BEM nodes neighboring $\Gamma$ on $\Gamma_{f}$, such that

$$
0>x_{1}=0.5 x_{2} .
$$

Then we use three points $\left(x_{1}, \eta_{2}^{n+1}\left(x_{1}\right),\left(x_{2}, \eta_{2}^{n+1}\left(x_{2}\right)\right.\right.$ and $\left(-2 x_{1}, \eta_{1}^{n}\left(-2 x_{1}\right)\right)$ as the nodal values defining a quadratic Lagrange polynomial $\eta_{I}(x)$, and assign

$$
\eta^{n+1}(0) \triangleq \eta_{I}(0)
$$

We do not use higher-order interpolations because the free surface in the PF and NSE subdomain is tracked/captured using cubic splines and level-set method based on linear elements, respectively. Thus it is not necessary to attempt orders higher than cubic. There are other interpolations that are in general more preferred in numerical analysis, such as using Chebyshev nodes [63]. However, without a priori knowledge of the wave profile near $\Gamma$, we use symmetric interpolation interval that contains $x=0$. The quadratic Chebyshev nodes for this interval requires data at $x=0$, which is exactly what we try to recover.

To estimate the above interpolation, recall the interpolation error [63]

$$
\eta(x)-\eta_{I}(x)=\frac{1}{(n+1) !} \eta^{(n+1)}(\zeta(x)) \prod_{i=0}^{n}\left(x-x_{i}\right),
$$

with $\left(x_{i}, \eta_{i}\right)$ being the data, $\zeta$ in the interpolation interval and $n=2$ in our case. Since solitary waves are often used in the coastal and offshore engineering community to study large wave (such as tsunami) impact, here we apply Eq. (11) to the $\operatorname{sech}^{2}$ soliton solution

$$
\eta(x, t)=H \operatorname{sech}^{2}(k(x-C t))
$$

of the Korteweg-de Vries (KdV) equation [64]

$$
\eta_{\tau}+3 \eta \eta_{\xi}+\frac{1}{3} \eta_{\xi \xi \xi}=0
$$

Here $\tau$ and $\xi$ denote proper time and space scale, respectively. The long waves described by the KdV equation (13) have the exact counteracting dispersiveness and nonlinearity effects, therefore can maintain their profiles for a long duration. In Eq. (12) $C$ is the wave celerity and $k=\sqrt{3 H /\left(4 h^{3}\right)}$. We consider the scenario when the crest is at the interface, thus $t=0$. For the 3 rd-order derivative of $\operatorname{sech}^{2}(x)$ near $x=0$, we have

$$
\left|\left(\operatorname{sech}^{2}(x)\right)^{(3)}\right|<5
$$

near $x=0$. By Eq. (11) we have

$$
\frac{\left|\eta(x)-\eta_{I}(x)\right|}{H}<\frac{5}{6} k^{3}\left|\prod_{i=0}^{n}\left(x-x_{i}\right)\right| .
$$


Recall that the interpolation data for $\eta_{I}$ is chosen according to the boundary element size near $x=0$, let $h_{b}$ the characteristic element size (in $x$ direction), then Eq. (14) becomes

$$
\frac{\left|\eta(x)-\eta_{I}(x)\right|}{H}<\frac{15}{24} \sqrt{\frac{3}{4}} \sqrt{\frac{H}{h}}\left(\frac{x}{h}-\frac{h_{b}}{h}\right)\left(\frac{x}{h}+\frac{h_{b}}{h}\right)\left(\frac{x}{h}+2 \frac{h_{b}}{h}\right) .
$$

In the BEM discretization when the soliton is at the interface we usually have $h_{b} / h \leq 0.3$. The error estimation in Eq. (15) near $x=0$ is shown in Figure 3, with different (normalized) wave height $H / h$ and element size $h_{b} / h$. The error increases as the wave height and element size increases, as shown in Figure 4 for the location of crest $(x=0)$. In general, near the interface $(x / h \leq 0.3)$, the error of the free surface reconstruction is less than $3 \%$. This is within acceptable margin of most coastal and offshore engineering applications.

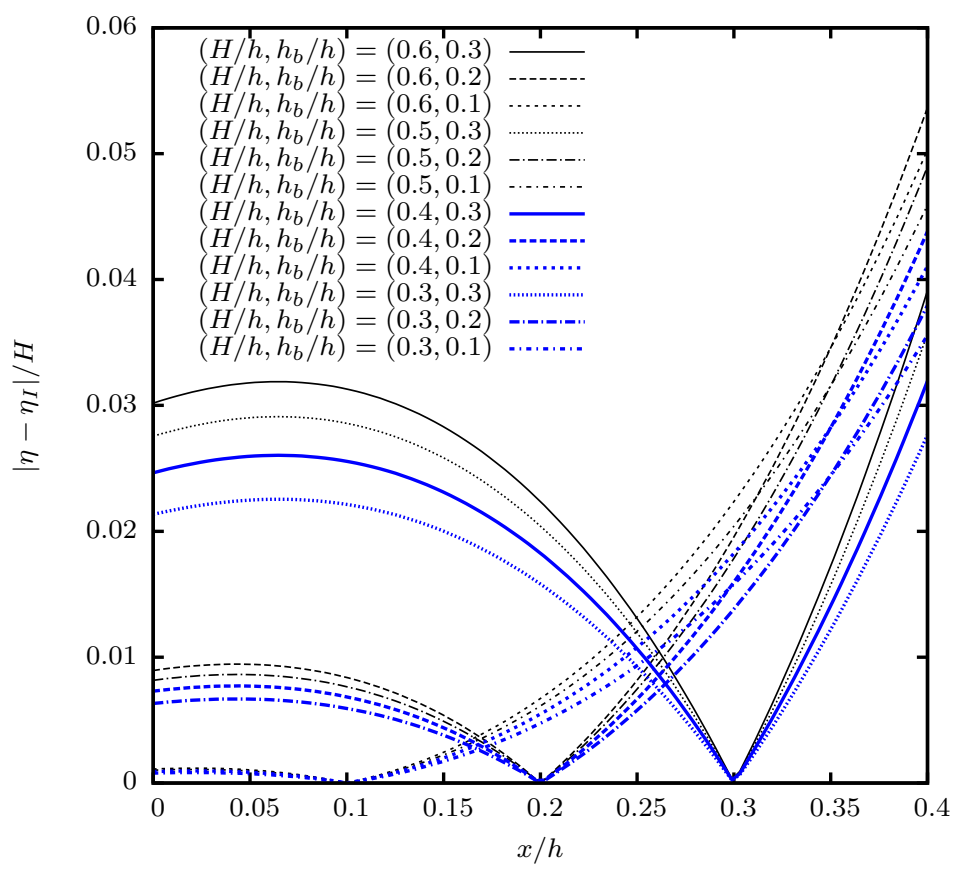

Figure 3: The error of free surface interpolation for various wave height $H / h$ and boundary element size $h_{b} / h$ near the interface.

However, the estimation above does not account for the staggered stepping. Figure 5 shows the interpolated free surface profile for $H / h=0.6$ and $h_{b} / h=0.2$. $\eta_{I}^{*}$ is from the interpolation in which the data in $\Omega_{\mathrm{NSE}}$ is from the wave profile $\eta$ at previous time step $(\Delta t=0.005)$. One can see a phase delay from this staggered time stepping. Of course in simulations the error introduced near the interface is also contributed by other sources, such interpolation the error through nonconforming mesh. Their effect remains to be studied.

\section{Interpretation of the DD method by Helmholtz decomposition}

In this section we present the mathematical interpretation of the coupling scheme, thus adding the robustness of the method. This could also be explored for future improvement of the scheme. The interpretation is based on the Helmholtz decomposition which says that a vector field can be decomposed into a solenoidal and a curl-free component. Here we only consider the case of bounded domains, and the result can be stated as 

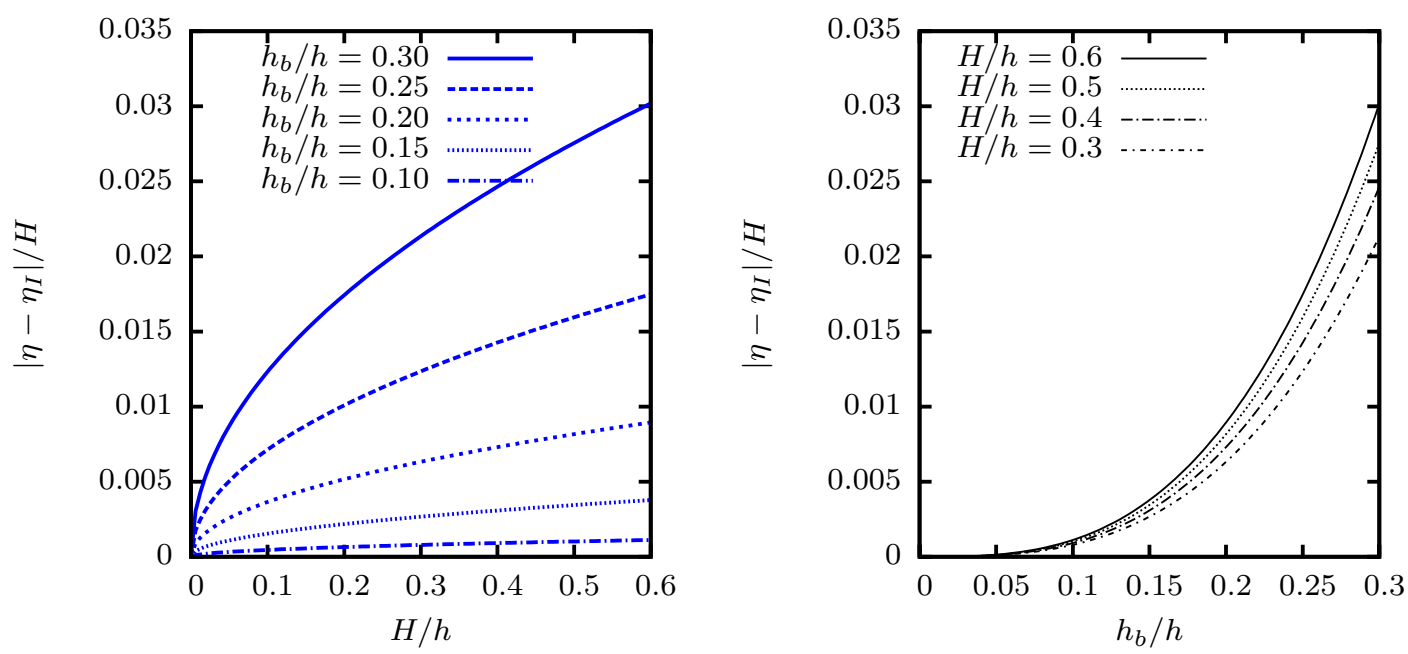

Figure 4: The error at the wave crest $x=0$ of free surface interpolation.

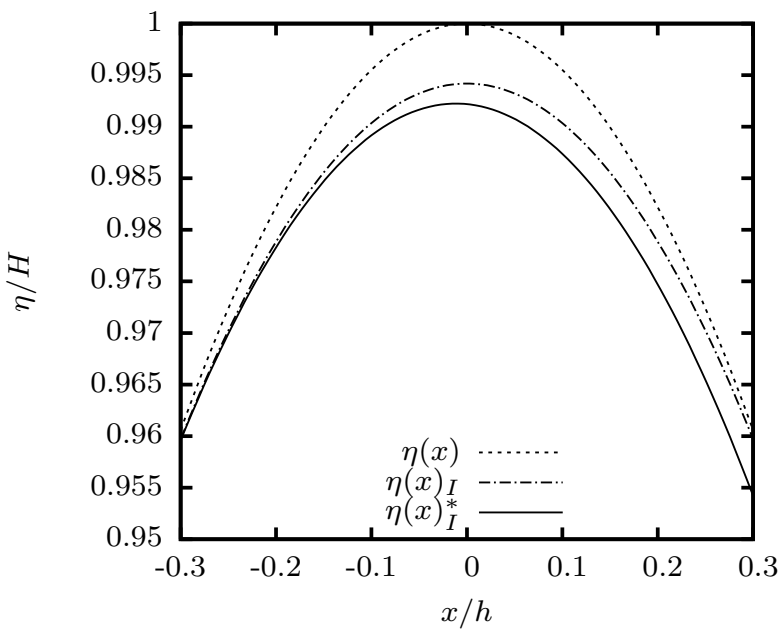

Figure 5: The $\operatorname{sech}^{2}$ solitary wave profile $\eta$ near $x=0$, with $H / h=0.6$. The interpolation $\eta_{I}$ is from $h_{b} / h=0.2$. $\eta_{I}^{*}$ is from using $\eta$ from $\Delta t=0.005$ ago for the point data in $\Omega_{\mathrm{NSE}}$. The staggered interpolation of the right-running wave gives a phase shift. 
Theorem 4.1 ([52, §1.2.2], [65, Lemma 2.5.1 \& 2.5.3, II] $]$ ). For a bounded, connected Lipschitz domain $\Omega$ with boundary $\partial \Omega, \boldsymbol{L}^{2}(\Omega)$ has the decomposition

$$
\boldsymbol{L}^{2}(\Omega)=L_{\sigma}^{2}(\Omega) \oplus G(\Omega),
$$

with

$$
L_{\sigma}^{2}(\Omega)=\left\{\boldsymbol{v} \in \boldsymbol{L}^{2}(\Omega) ; \nabla \cdot v=0,\left.\boldsymbol{n} \cdot \boldsymbol{v}\right|_{\partial \Omega}=0\right\}
$$

and

$$
G(\Omega)=\left\{\boldsymbol{w} \in \boldsymbol{L}^{2}(\Omega) ; \exists p \in L^{2}(\Omega): \boldsymbol{w}=\nabla p\right\}
$$

being orthogonal in $\boldsymbol{L}^{2}(\Omega)$, i.e.,

$$
(\boldsymbol{v}, \boldsymbol{w})=0, \quad \forall \boldsymbol{v} \in L_{\sigma}^{2}(\Omega), \boldsymbol{w} \in G(\Omega) .
$$

Here $(\cdot, \cdot)$ denotes the inner product in $\boldsymbol{L}^{2}(\Omega)$.

In Eq. (17)-(18) the derivatives of $\nabla$. and $\nabla$ operators are in the distribution sense. Theorem 4.1 implies a projection operator $\mathcal{P}: \boldsymbol{L}^{2}(\Omega) \rightarrow L_{\sigma}^{2}(\Omega)$, such that $\mathcal{P}$ projects an element of $\boldsymbol{L}^{2}(\Omega)$ to its solenoidal component. Let $\mathcal{I}$ be the identity operator, then we can define $\mathcal{P}_{\perp}: \boldsymbol{L}^{2}(\Omega) \rightarrow G(\Omega)$, such that $\mathcal{P}_{\perp}=\mathcal{I}-\mathcal{P}$. Then Theorem 4.1 says that for any $\boldsymbol{u} \in \boldsymbol{L}^{2}(\Omega)$, uniquely we have

$$
\boldsymbol{u}=\mathcal{P}(\boldsymbol{u})+\mathcal{P}_{\perp}(\boldsymbol{u})
$$

Recall that on $\Omega_{\mathrm{PF}}$ the mass and momentum conservation laws are reduced to Eq. (1d) and Bernoulli's equation, respectively. By solving them on $\Omega_{\mathrm{PF}}$, one hopes to recover $\nabla \varphi$ as an approximation of $\mathcal{P}_{\perp}$. However, in general $\mathcal{P}_{\perp}$ does not satisfy Bernoulli's equation, that is,

$$
\frac{\partial \mathcal{P}_{\perp}(\boldsymbol{u})}{\partial t}+\frac{1}{2} \nabla\left|\mathcal{P}_{\perp}(\boldsymbol{u})\right|^{2}+\frac{\nabla p}{\rho}+\frac{\boldsymbol{f}}{\rho}=\mathbf{0}
$$

is not always true, so what is the counterpart of Bernoulli's equation for $\mathcal{P}_{\perp}(\boldsymbol{u})$ ? To answer this, let $\varphi$ be the solution of Eq. (1d) with Neumann boundary data $\boldsymbol{u}^{*} \cdot \boldsymbol{n}$ on $\partial \Omega_{\mathrm{PF}} \backslash \Gamma$ and $\boldsymbol{u} \cdot \boldsymbol{n}$ on $\Gamma$, thus [66]

$$
\frac{\partial \mathcal{P}_{\perp}(\boldsymbol{u})}{\partial t}+\frac{1}{2}\left|\mathcal{P}_{\perp}(\boldsymbol{u})\right|^{2}+\frac{\nabla p}{\rho}+\frac{f}{\rho}=\xi(\boldsymbol{u}),
$$

with

$$
\xi(\boldsymbol{u})=\nu \nabla^{2} \mathcal{P}(\boldsymbol{u})-\frac{\partial \mathcal{P}(\boldsymbol{u})}{\partial t}-\nabla \cdot\left(\mathcal{P}(\boldsymbol{u}) \otimes \mathcal{P}_{\perp}(\boldsymbol{u})+\mathcal{P}_{\perp}(\boldsymbol{u}) \otimes \mathcal{P}(\boldsymbol{u})+\mathcal{P}(\boldsymbol{u}) \otimes \mathcal{P}(\boldsymbol{u})\right) .
$$

By Eq. (22), $\varphi$ does not satisfy Bernoulli's equation with pressure $p$. As pointed out by Joseph [66], "there may be hidden irrotational terms on the right-hand side of this equation". In fact, the projection $\mathcal{P}_{\perp}(\xi(\boldsymbol{u}))$ can be moved to the left hand side and considered as an additional pressure contribution, as in

$$
\frac{\partial \mathcal{P}_{\perp}(\boldsymbol{u})}{\partial t}+\frac{1}{2} \nabla\left|\mathcal{P}_{\perp}(\boldsymbol{u})\right|^{2}+\left(\frac{\nabla p}{\rho}-\mathcal{P}_{\perp}(\xi(\boldsymbol{u}))\right)+\frac{\boldsymbol{f}}{\rho}=\mathcal{P}(\xi(\boldsymbol{u})) .
$$

If we can further assume negligible $\mathcal{P}(\boldsymbol{u})$, then

$$
\frac{\partial \mathcal{P}_{\perp}(\boldsymbol{u})}{\partial t}+\frac{1}{2} \nabla\left|\mathcal{P}_{\perp}(\boldsymbol{u})\right|^{2}+\left(\frac{\nabla p}{\rho}-\mathcal{P}_{\perp}(\xi(\boldsymbol{u}))\right)+\frac{\boldsymbol{f}}{\rho} \approx \mathbf{0} .
$$

That is, if a Bernoulli-like formulation for $\mathcal{P}_{\perp}(\boldsymbol{u})$ is expected, at least the pressure component should be modified to incorporate the effect of viscosity and vorticity. Neglecting this additional pressure component is another form of the modeling error. 
Now we interpret the matching conditions (1h)-(1i) with the Helmholtz projection. It is easy to see that Eq. (1h) is equivalent to

$$
\mathcal{P}_{\perp}(\boldsymbol{u})=\boldsymbol{u}
$$

and Eq. (1i) is equivalent to Eq. (21), upon an additive constant. In fact, Eq. (25) and (21) can be seen as "strong" and "weak" statements of the flow irrotationality, respectively. This is because Eq. (25) gives $\mathcal{P}(\boldsymbol{u})=\mathbf{0}$, which further indicates $\xi(\boldsymbol{u})=\mathbf{0}$ in Eq. (22). Thus Eq. (22) becomes Eq. (21). On the other hand, introducing $(21)$ into $(22)$ only gives $\xi(\boldsymbol{u})=\mathbf{0}$. Therefore, both matching conditions enforce the Helmholtz projection in some sense. It should be pointed out that these two matching conditions at $\Gamma$ do not imply the flow in $\Omega_{\mathrm{PF}}$ is irrotational. The potential flow model must be assumed a priori. However, when the flow in $\Omega_{\mathrm{PF}}$ is indeed irrotational, with the Neumann boundary data on $\partial \Omega_{\mathrm{PF}} \backslash \Gamma$ and matching condition (25), well-posedness of the Laplace equation on a Lipschitz domain guarantees that Helmholtz projection produces the potential flow velocity.

Now let us interpret operator $\mathcal{M}$ and $\mathcal{N}$ introduced in the previous section. It was pointed out in [42] that $\mathcal{M}$ is an extension of the Poincaré-Steklov operator. To substantiate this statement, we use standard Sobolev space $H^{1 / 2}(\Gamma)$ and $H^{-1 / 2}(\Gamma)$. Then the Poincaré-Steklov operator for the Laplace problem on $\Omega_{\mathrm{PF}}$ is defined as $\mathcal{T}: H^{1 / 2}(\Gamma) \rightarrow H^{-1 / 2}(\Gamma)$ such that

$$
\mathcal{T}: \lambda \rightarrow \gamma_{1} \varphi_{\Gamma}, \forall \lambda \in H^{1 / 2}(\Gamma)
$$

where $\varphi$ solves (1d) with corresponding Neumann boundary condition on $\partial \Omega_{\mathrm{PF}} \backslash \Gamma$ and Dirichlet boundary condition $\varphi=\lambda$ on $\Gamma$, and $\gamma_{1}$ denotes the trace operator. It is known [57] that $\mathcal{T}$ is continuous and has inverse $\mathcal{S}=\mathcal{T}^{-1}$ such that

$$
\mathcal{S}: H^{-1 / 2}(\Gamma) \rightarrow H^{1 / 2}(\Gamma) .
$$

That is, operator $\mathcal{S}$ receives a Neumann boundary datum on $\Gamma$ and returns $\varphi$ for the solution of the same boundary value problem as in the definition of $\mathcal{T}$. $\mathcal{S}$ is called the Steklov-Poincaré operator. With respect to their behavior, $\mathcal{T}$ and $\mathcal{S}$ are also referred to as the Dirichlet-Neumann operator and the Neumann-Dirichlet operator, respectively. Thus, we see that for a given $\varphi \in H^{1 / 2}(\Gamma)$, the normal component of $\mathcal{M}(\varphi)$ is $\mathcal{T}(\varphi)$. The tangential component(s) can be obtained directly from $\varphi$ on $\Gamma$, without resorting to solving Eq. (1d). On the other hand, we notice that for a given velocity field $\boldsymbol{u} \in \boldsymbol{L}^{2}(\Omega)$, we have

$$
\left.\mathcal{P}_{\perp}(\boldsymbol{u})\right|_{\Gamma} \cdot \boldsymbol{n} \in H^{-1 / 2}(\Gamma),
$$

thus

$$
\mathcal{S}\left(\left.\mathcal{P}_{\perp}(\boldsymbol{u})\right|_{\Gamma} \cdot \boldsymbol{n}\right) \in H^{1 / 2}(\Gamma) .
$$

Therefore, operator $\mathcal{M}$ and $\mathcal{S}\left(\mathcal{P}_{\perp}(\cdot) \cdot \boldsymbol{n}\right)$ can be considered as the inverse of each other in some sense, between space $\boldsymbol{L}^{2}(\Gamma)$ and $H^{1 / 2}(\Gamma)$. The problem is that $\mathcal{P}_{\perp}(\cdot)$ is not $1-1$. Thus the solenoidal component $\mathcal{P}(\boldsymbol{u})$ is lost and can not be recovered by $\mathcal{M}$. Instead, one can see that the range of $\mathcal{M}$ is a subset of $\gamma_{1}\left(\boldsymbol{L}^{2}(\Gamma)\right)$. In fact, we have

$$
\left.\mathcal{M}\left(H^{1 / 2}(\Gamma)\right) \subset \gamma_{1}(G(\Omega))\right|_{\Gamma}
$$

In other words, applying $\mathcal{M}$ does not return us to the original space $\boldsymbol{L}^{2}(\Gamma)$ but to its irrotational subset. This loss of information is exactly remedied by the matching condition $(25)$, which says $\left.\gamma_{1}(G(\Omega))\right|_{\Gamma}=\left.\gamma_{1}\left(\boldsymbol{L}^{2}(\Omega)\right)\right|_{\Gamma}$. Thus, another interpretation of the matching condition (25) is that it provides a sufficient condition for $\mathcal{M}$ to be the exact the inverse of the composition of $\mathcal{P}_{\perp}$ and the Steklov-Poincare operator.

The interpretation of operator $\mathcal{N}$ is similar, when we consider the Pressure Poisson Equation (PPE)

$$
\nabla^{2} p=\rho \nabla \cdot\left(-\boldsymbol{u} \cdot \nabla \boldsymbol{u}+\nu \nabla^{2} \boldsymbol{u}\right)
$$

for the pressure $p$ in an incompressible flow. Eq. (28) is obtained by applying $\nabla$. to (1a) and incorporating (1b). The Neumann boundary condition for (28) can also be obtained by taking the normal component of (1a) on the boundary. Then we immediately see that $\mathcal{N}$ incorporates the Steklov-Poincaré operator for the Poisson problem (28), with the Neumann data obtained by solving the NSE with given $\boldsymbol{u}$ on $\Gamma$. 


\section{Numerical examples}

In this section, we present two numerical examples to validate the 3D heterogeneous DD model. In the first one we model a dambreak wave impacting a fixed rigid body at downstream. In the second example a solitary wave impacting an array of surface-piercing cylinders is simulated. In both examples numerical results are compared with experimental data. We also present the wall-time results for each case to demonstrate the efficiency of the method. The fluid (water) is assigned with density $\rho=1.0 \times 10^{3} \mathrm{~kg} / \mathrm{m}^{3}$ and viscosity $\mu=1.0 \times 10^{-3} \mathrm{~Pa} \cdot \mathrm{s}$. The acceleration of gravity is $g=9.806 \mathrm{~m} / \mathrm{s}^{2}$. The coupled model is implemented on a Linux workstation with 12 Intel ${ }^{\circledR}$ Xeon ${ }^{\circledR}$ X5660 (2.80GHz) CPUs, and 190 GB RAM.

\subsection{Dam break wave impact obstacle}

In this example we demonstrate the basic capability of the DD method by examining the initial stage of a dam-break wave impacting a fixed rigid structure downstream. The dambreak wave is a canonical test example in the offshore and coastal engineering community, due to its easy setup, rich content in wave phenomena and well-studied wave-breaking behavior. As shown in Figure 6, the reservoir is mostly modeled using the PF, and the downstream is modeled by the NSE, thus the downstream flow domain belongs to $\Omega_{\mathrm{NSE}}$, and the reservoir is occupied by $\Omega_{\mathrm{PF}}$ and part of $\Omega_{\mathrm{NSE}}$.
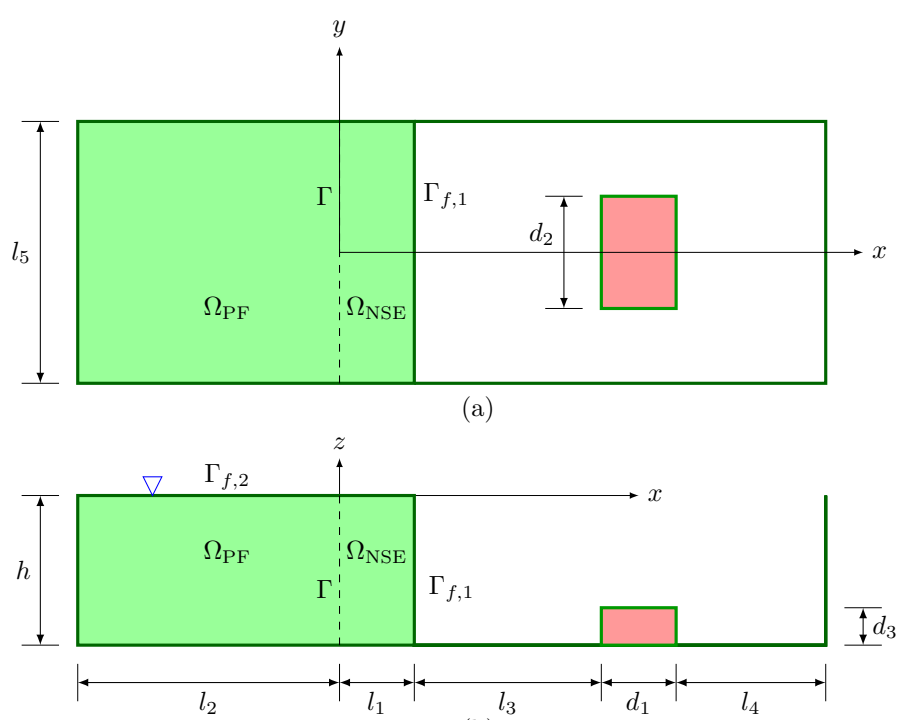

(a)

(b)

\begin{tabular}{cc}
\hline$h$ & $0.550 \mathrm{~m}$ \\
$d_{1}$ & $0.160 \mathrm{~m}$ \\
$d_{2}$ & $0.400 \mathrm{~m}$ \\
$d_{3}$ & $0.160 \mathrm{~m}$ \\
$l_{1}$ & $0.220 \mathrm{~m}$ \\
$l_{2}$ & $1.000 \mathrm{~m}$ \\
$l_{3}$ & $1.170 \mathrm{~m}$ \\
$l_{4}$ & $0.670 \mathrm{~m}$ \\
$l_{5}$ & $1.000 \mathrm{~m}$ \\
\hline
\end{tabular}

Figure 6: Geometric configurations of the dambreak wave impact: (a) plan view and (b) side view.

The unstructured grid of $\Omega_{\mathrm{NSE}}$ is generated using Gmsh [67] which controls the mesh size through characteristic length $l c_{\boldsymbol{x}}$ at multiple key points $\boldsymbol{x}$ (the sizes of elements are determined through linear interpolation). The structured grid of $\Omega_{\mathrm{PF}}$ is generated and updated by the BEM solver, with $M_{x}, M_{y}$ and $M_{y}$ as the number of elements in each coordinate direction. Three sets of meshes are used to test the effect of interface meshing on model convergence, as shown in Table 1. Other mesh information remain the same in all three tests, as $M_{x}=15, l c=0.005 \mathrm{~m}$ at the dam and $l c=0.005 \mathrm{~m}$ at the obstacle surface. A side view of the meshed domain is shown in Figure 7. All boundaries are imposed with a free-slip condition. A fixed time step of $\Delta t=0.005 \mathrm{~s}$ is used.

When the simulation starts, the free surface $\Gamma_{f, 1}$ transforms into a wave front, while the elevation $\Gamma_{f, 2}$ decreases as fluid flows away from $\Omega_{\mathrm{PF}}$ into $\Omega_{\mathrm{NSE}}$. An impact happens when the wave front reaches the downstream obstacle. Figure 8 shows snapshots of the wave front in $\Omega_{\mathrm{NSE}}$ at the moment of impact. The level set free surface tracking successfully captures the complex wave front including the jet generation. The 


\begin{tabular}{ccccc}
\hline & & & \multicolumn{2}{c}{$l c_{\boldsymbol{x}}(\mathrm{m})$} \\
\cline { 4 - 5 } Job ID & $M_{y}$ & $M_{z}$ & $\boldsymbol{x}=(0,0,-h)$ & $\boldsymbol{x}=(0,0,0)$ \\
\hline DD1 & 5 & 5 & 0.100 & 0.020 \\
DD2 & 10 & 6 & 0.050 & 0.010 \\
DD3 & 10 & 6 & 0.025 & 0.005 \\
\hline
\end{tabular}

Table 1: Mesh information at the interface $(x=0)$.

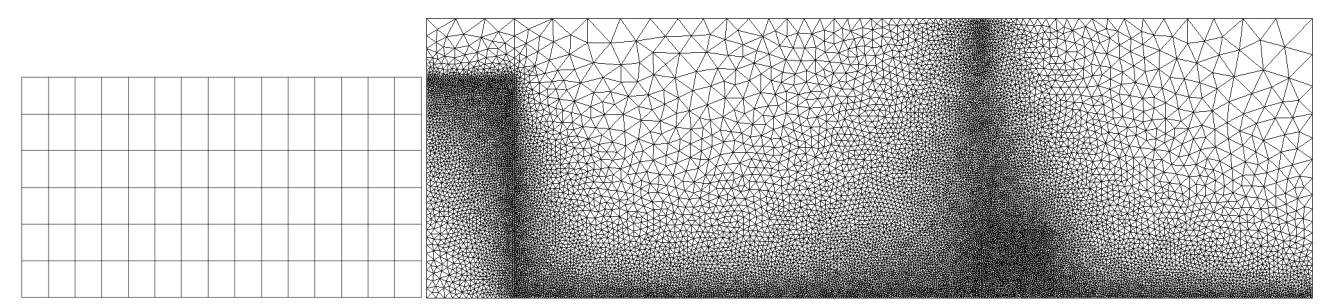

Figure 7: Side view of the meshed subdomains.
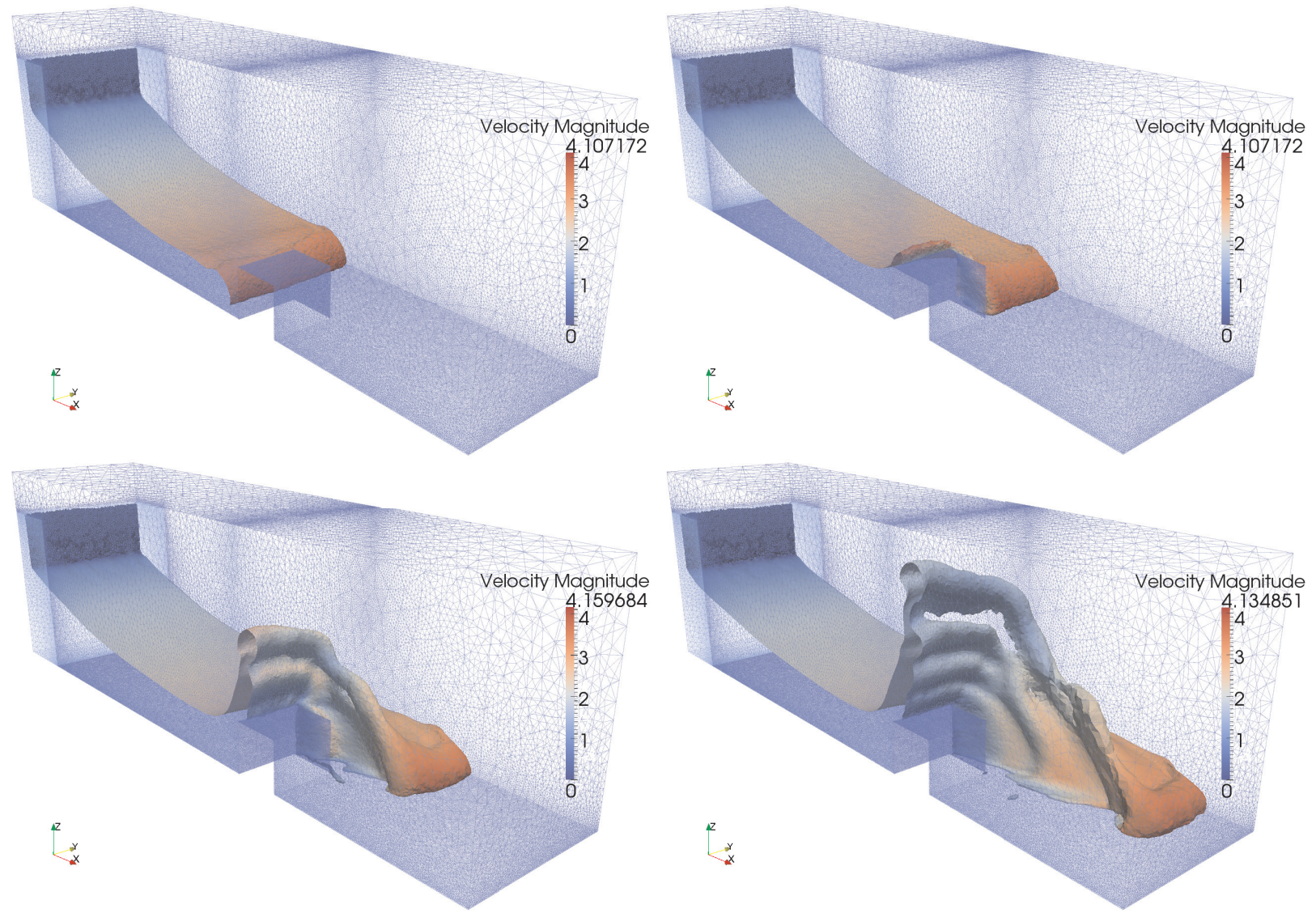

Figure 8: Free surface profile in $\Omega_{\mathrm{NSE}}$ overlaid with velocity magnitude $(\mathrm{m} / \mathrm{s})$ at the initial impact stage. Snapshots only show half $(y>0)$ of the subdomain about the $x-z$ symmetry plane. The background shows the mesh of $\Omega_{\mathrm{NSE}}$. From left-up to right-bottom: $t(g / h)^{1 / 2}=2.11, t(g / h)^{1 / 2}=2.53, t(g / h)^{1 / 2}=2.96, t(g / h)^{1 / 2}=3.38$. At the upstream (left) end of each snapshot, the dark area indicates the part of the interface $\Gamma$ that was originally wet but now is above the free surface because of dambreak. 
decrease (dark area) of the free surface at the upstream (left) end of the NSE subdomain in each snapshot indicates the elevation change at the interface.

Figure 9 shows the time history of the free surface elevation from our DD simulation at two locations, within the PF subdomain $\Omega_{\mathrm{PF}}$ and the NSE subdomain $\Omega_{\mathrm{NSE}}$, respectively. The results are compared with those from previous experiment and numerical simulation by Kleefsman et al. [1]. Their numerical simulation was performed using a viscous flow solver (COMFLOW) for the entire domain $\Omega_{\mathrm{NSE}} \cup \Omega_{\mathrm{PF}}$. The general agreement observed between the DD prediction and the results from [1] indicates an accurate prediction of the flow behavior across the coupling interface. Notice that the DD results show a greater rate of free surface rising when the wave front arrives $\left(t(g / h)^{1 / 2} \approx 2\right)$, this is due to a thicker and more uniform front produced by the level-set technique, as can be seen from Figure 8. However, other than the higher rate of surface rising, the results of DD method match those from [1] faithfully.
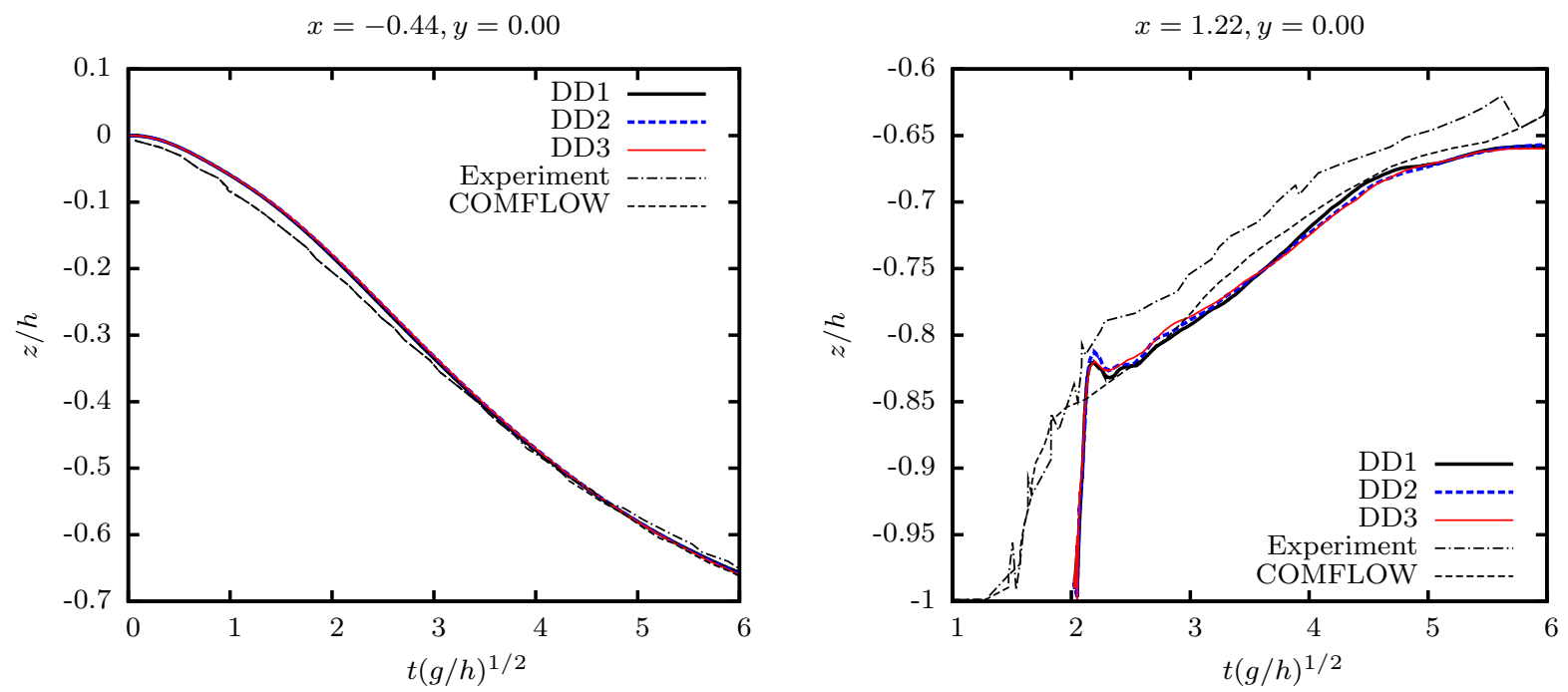

Figure 9: Free surface elevation time history during dambreak, at locations within $\Omega_{\mathrm{PF}}$ (left) and $\Omega_{\mathrm{NSE}}$ (right).

Figure 10 shows a comparison of the pressure on the windward face $\left(x=l_{1}+l_{3}\right)$ of the obstacle. For pressure results at $z=-0.525 \mathrm{~m}$, both the peak value and the subsequent quasi-static state match well. For results at $z=-0.451(\mathrm{~m})$, one can see that the DD result is very close to the COMFLOW simulation result, especially the quasi-static state, and both numerical solvers produce lower values than the experiment, though the DD simulation predicts the peak pressure better.

The effect of a refined mesh at the interface is studied by examining the pressure at the above two locations in Figure 10. Let $h_{e}$ be the characteristic size of the finite element mesh of $\Gamma$ in DD1, then DD2 has a characteristic size $2 h_{e}$ and DD3 has $4 h_{e}$. The relative error between the DD solution $p_{h_{e}}$ and the experiment results $p_{\text {exp }}$ are shown in Figure 11. Though the error decreases along with mesh refinement, it is difficult to characterize their relation without further analysis of the experiment error and the interplay of factors that are affected by the interface mesh size, such as the nonconforming mesh interpolation and free surface elevation reconstruction.

To study the computing cost of the DD method, a finite element simulation in which the entire domain is modeled by the NSE (thus $\Omega_{\mathrm{PF}}=\emptyset$ and $\Omega=\Omega_{\mathrm{NSE}}$ ) is performed, with the mesh adopting the same characteristic length at key points as the FEM mesh in DD1 (see Table 1). Let $T_{w}$ be the wall time of each DD simulation, and $T_{w 0}$ the wall time of the finite element simulation, Figure 12 shows the accumulated wall time cost (normalized by $T_{w 0}$ ) of each subdomain solver in the first 70 time steps. It can be seen that the BEM solver costs only a small fraction of the time (less than 5\%) of the FEM solver. As to the combined wall time (FEM+BEM), DD1 and DD2 are 10\% and 5\% less than FEM, respectively. Due to a much refined mesh in DD3, the total wall time is greater than that of the FEM simulation. Thus we can observe for this 

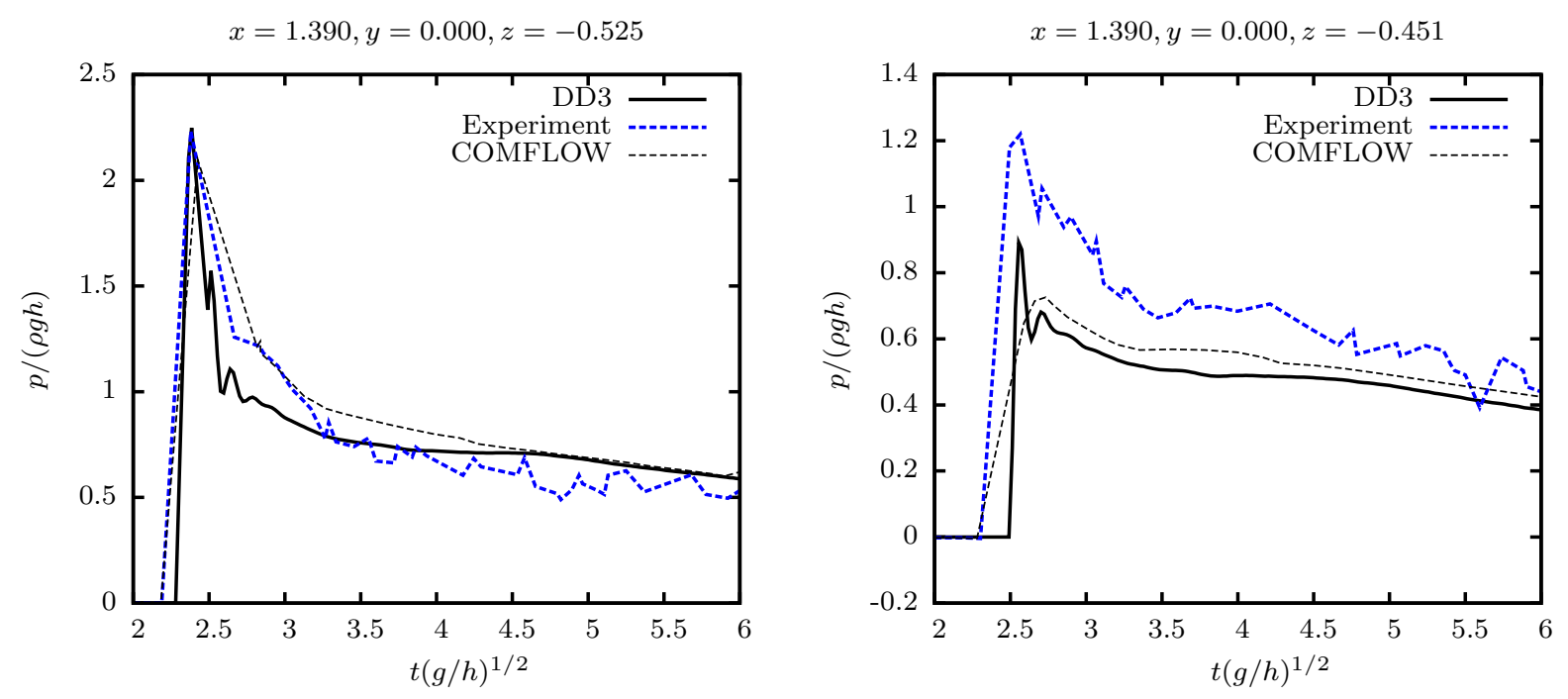

Figure 10: Time history of the pressure on the impact face of the obstacle.
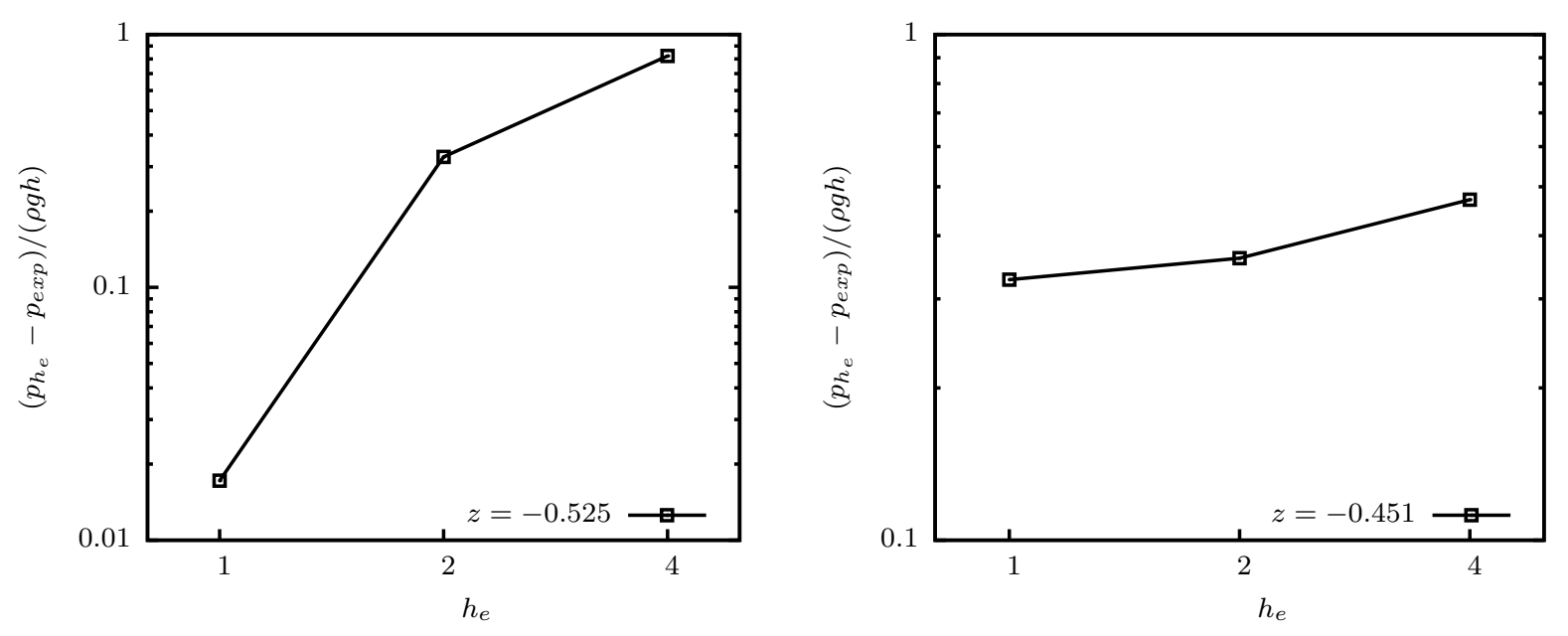

Figure 11: Normalized impact pressure errors at $z=-0.525$ (left) and $z=-0.451$ (right). 


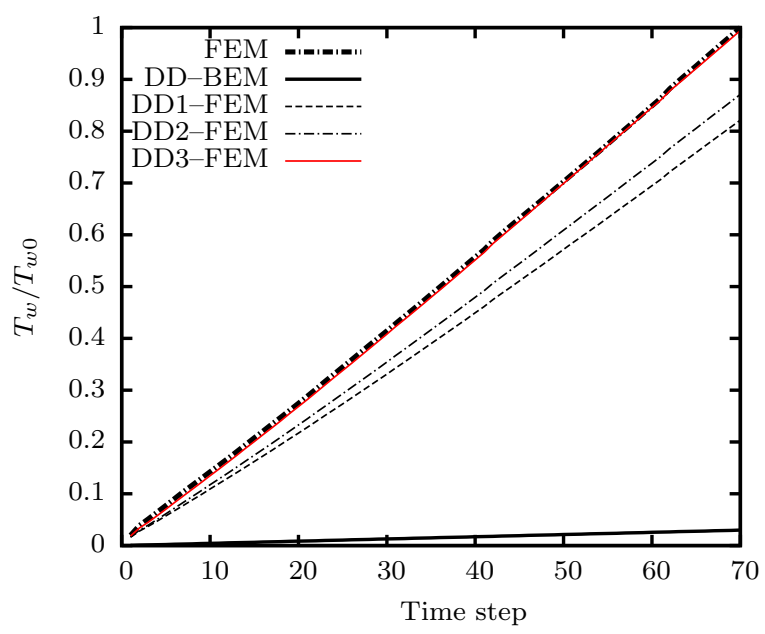

Figure 12: Wall time for dambreak impact simulation from DD and FEM models.

example that the FEM cost in $\Omega_{\mathrm{NSE}}$ dominates the wall time, due to the small size of $\Omega_{\mathrm{PF}}$ among $\Omega$ (see Figure 7) and the relatively simple free surface evolution of the PF subdomain. However, the effect of the DD method in reducing the total computing cost is obvious.

\subsection{Solitary wave impact cylinders}

Wave impact on cylinders has been actively studied in the past several decades due to the wide application of the structural form in coastal and offshore engineering, such as bridge columns, drilling risers and jacket platforms. Solitary waves are often used by coastal and offshore engineers to model tsunami, a major hazard in coastal regions. In this example we apply the DD method to simulate the impact of a solitary wave on a cylinder array.

The geometry of the test environment is shown in Figure. 13. The wave is generated in $\Omega_{\mathrm{PF}}$, and an array consisting of three cylinders is situated downstream. The theory of wave making and its validation of the BEM solver is discussed by Grilli and coworkers in $[10,13,44]$. The solitary wave has a height of $H=0.6 h$. The numerical results are compared with those from an experiment [68]. During the experiment, the cylinder at $l_{1}+l_{3}$ is instrumented and is referred to as the "instrumented" cylinder. The other two are referred to as "dummy" cylinders as they serve as flow obstacles. A nonslip boundary is imposed at the wall of cylinders. To study the effect of time step for our explicit DD scheme, several simulations are performed with $\Delta t=0.005 \mathrm{~s}$, $0.01 \mathrm{~s}$ and $0.02 \mathrm{~s}$, labeled in the following as DD1, DD2 and DD3, respectively. Table 2 shows $l c$ at key points in the mesh of $\Omega_{\mathrm{NSE}}$. For the boundary mesh of $\partial \Omega_{\mathrm{PF}}$, we have $M_{x}=40, M_{y}=30$ and $M_{z}=6$.

\begin{tabular}{cc|cc}
\hline$x=0, z=-h$ & 0.10 & $x=0, z=0$ & 0.05 \\
$x=l_{1}+l_{2}+l_{4}, z=-h$ & 0.50 & $x=l_{1}+l_{2}+l_{4}, z=0$ & 0.20 \\
Cylinder wall, $z=-h$ & 0.10 & Cylinder wall, $z=0$ & 0.02 \\
\hline
\end{tabular}

Table 2: Characteristic length $(\mathrm{m})$ at key points for the mesh of $\Omega_{\mathrm{NSE}}$.

Figure 14 shows snapshots of the PF subdomain free surface profile. Only the positive $y$ half with respective to the symmetry plane $y=0$ are shown. The wave is generated (top left) and propagates (top right) in $\Omega_{\mathrm{PF}}$. Then it leaves through $\Gamma$ (middle row) and enters $\Omega_{\mathrm{NSE}}$ (now shown). The reflected and diffracted waves from the cylinder array propagating upstream can be seen at the bottom row of the snapshots. The time history of the free surface elevation at different locations is shown in Figure 15. Among them the first two locations are within $\Omega_{\mathrm{PF}}$, and the rest are within $\Omega_{\mathrm{NSE}}$. Observe that there is a general good 


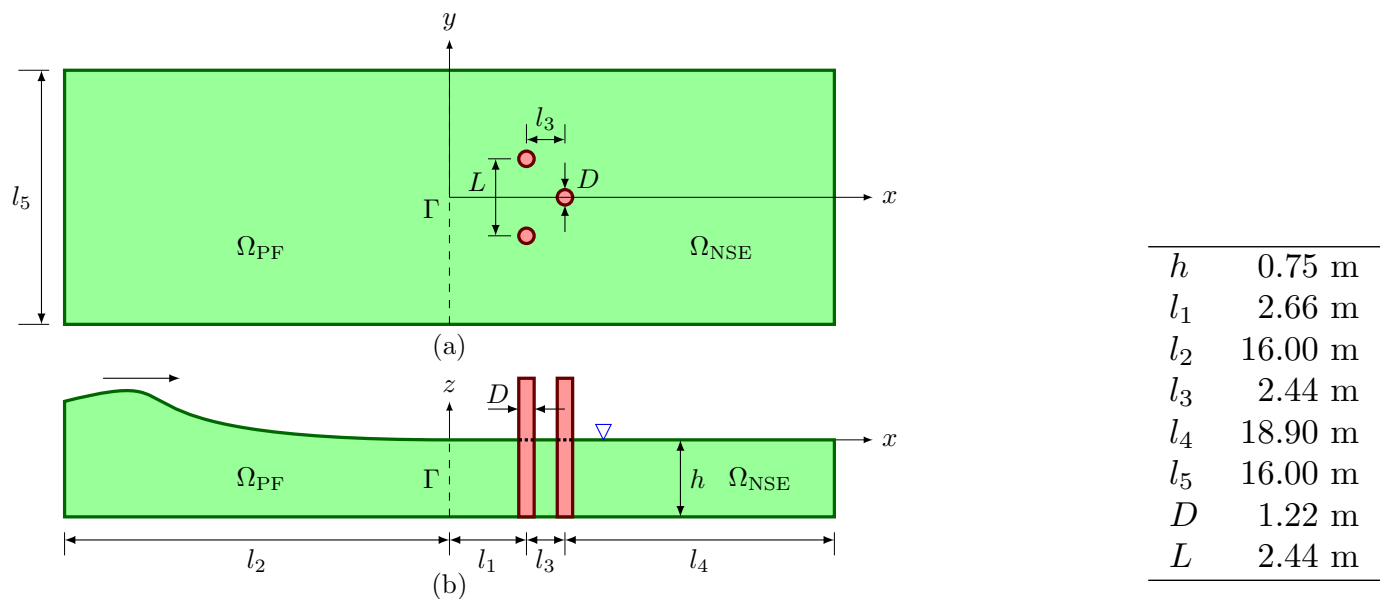

Figure 13: Geometric configurations of solitary wave impacting cylinders: (a) plan view and (b) side view.

agreement between the DD and experiment results. Notice a smaller wave height and a slight phase shift at locations in $\Omega_{\mathrm{NSE}}$, as both partially predicted by our analysis from the previous section. The wave heights at these locations are also used to study the temporal convergence. A simulation with a further reduced time step $\Delta t=0.0025 \mathrm{~s}$, denoted by DD0, is performed for comparison. Figure 16 shows a linear time-stepping error. This is consistent with the 1st-order scheme (equation (3)) used. The elevation history at the front of the instrumented cylinder $((x, y)=(4.22,0.00))$ from different time step is also shown. In particular, one can see the oscillations as an instability caused by large stepping in DD3. In terms of the free surface elevation, this instability is in a form of spurious waves.

Figure 17 shows the impact pressure time history at selected locations on the instrumented cylinder, with the phase shift in Figure 15 taken into account. One can notice a general agreement between DD and experiment results. These results show a uniform amount of pressure increase when the wave crest arrives at the cylinder, with a normalized magnitude approximately 0.4 , or $40 \%$ of the hydrostatic pressure. This is consistent with the long wave nature of the soliton.

Figure 18 shows snapshots of the free surface profile near the cylinder array, overlaid with velocity magnitude. One can see the vortex shedding at the cylinder boundary as the wave passes through, as well as a reflected wave propagating upstream. The last two snapshots show the wave pattern as the reflected wave propagates upstream and enters $\Omega_{\mathrm{PF}}$.

The total drag on the instrumented cylinder is shown in Figure 19. There is a good agreement, with DD results slightly less than that of the experiment, partially due to the smaller wave height in the numerical predictions. Compare the magnitude of the maximum positive and negative drag, with the latter mostly due to the hydrostatic force as the wave at the lee of the cylinder raises water height, notice that the dynamic effect is almost twice that of the hydrostatic force, contributing a large impact force on the cylinder.

Finally, let us study the efficiency of the DD method. Figure 20 shows wall time $\left(T_{w}\right)$ cost in each sub-problem, normalized by the final total wall time $\left(T_{w 0}\right)$ of the simulation. Notice that for each time step setting, the BEM solver costs much less, as little as less than $20 \%$ of the total time. Note also that this time cost ratio is not affected by time stepping, indicating a robustness in the coupling with respect to the computing efficiency. To further investigate the effect of the efficiency of the DD method, three numerical tests with different ratio $l_{\mathrm{NSE}} / l_{\mathrm{PF}}=\left(l_{1}+l_{3}+l_{4}\right) / l_{2}$ are conducted. In these tests, other geometric parameters are the same, and an identical time step of $t=0.01 \mathrm{~s}$ is used. To reduce the number of finite elements thus reduce the total computing time, in these tests the characteristic lengths for the finite element mesh are twice as those shown in Table 2 . The $l_{\mathrm{NSE}} / l_{\mathrm{PF}}$ ratio and the number of boundary elements in horizontal direction $M_{x}$ are shown in Table 3. Among these tests, case DD0 has the same geometry setting as in Figure 13, while in DD4 the PF subdomain size is reduced, and in DD5 it is reduced further, with the entire domain almost 

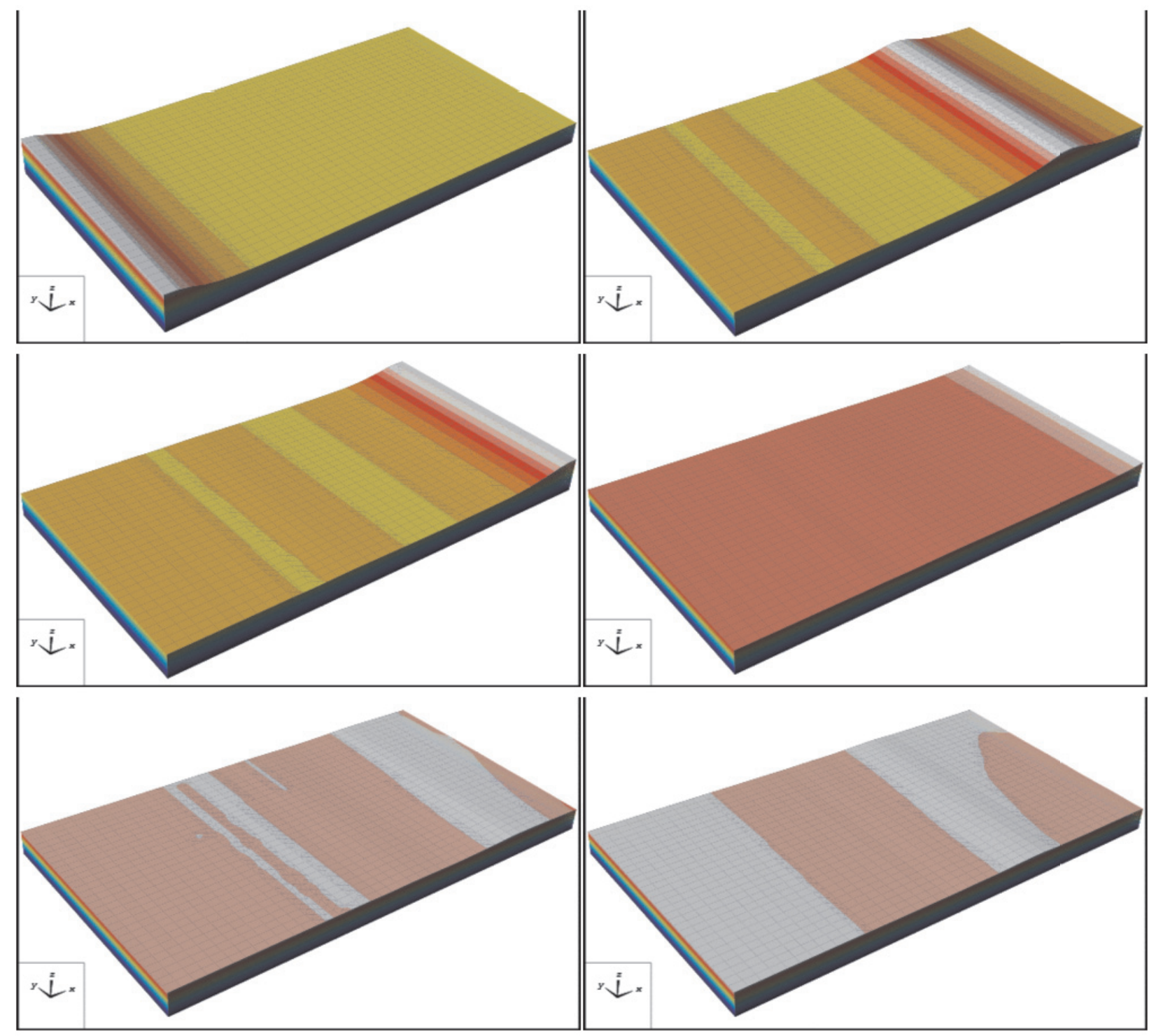

Figure 14: Free surface profiles in the PF subdomain $\Omega_{\mathrm{PF}}$. Color indicates normalized surface elevation. From left-top to right-bottom: $t(g / h)^{1 / 2}=5.4,18.1,21.7,23.5,30.7$ and 34.4.

\begin{tabular}{l|ll} 
& $l_{\mathrm{NSE}} / l_{\mathrm{PF}}$ & $M_{x}$ \\
\hline DD0 & $24 / 16$ & 40 \\
DD4 & $32 / 8$ & 20 \\
DD5 & $36 / 4$ & 10
\end{tabular}

Table 3: Geometry and element settings for tests approximating computing time. the number of boundary elements is reduced according as the horizontal length of the PF subdomain. The sum of subdomain length $l_{\mathrm{PF}}$ and $l_{\mathrm{NSE}}$ remains the same. 

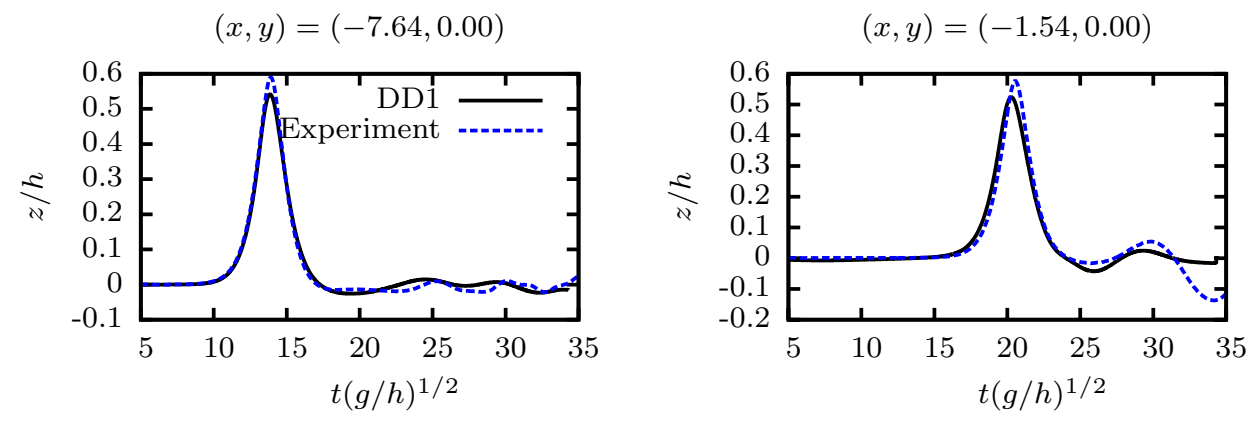

$(x, y)=(2.06,0.00)$
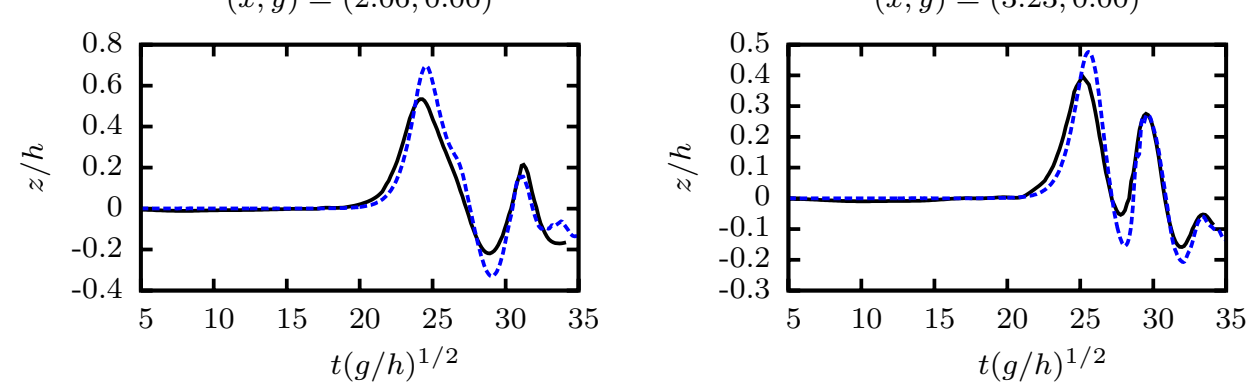

$(x, y)=(4.22,0.00)$

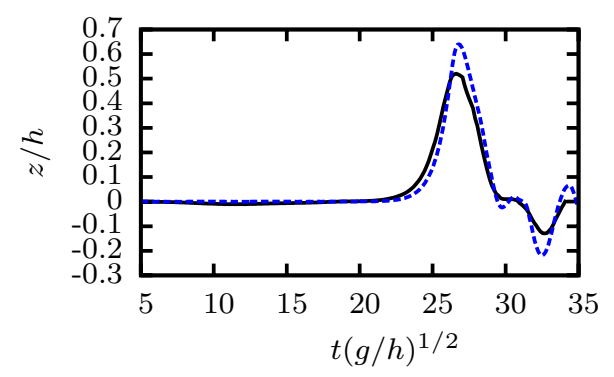

$(x, y)=(5.10,0.876)$

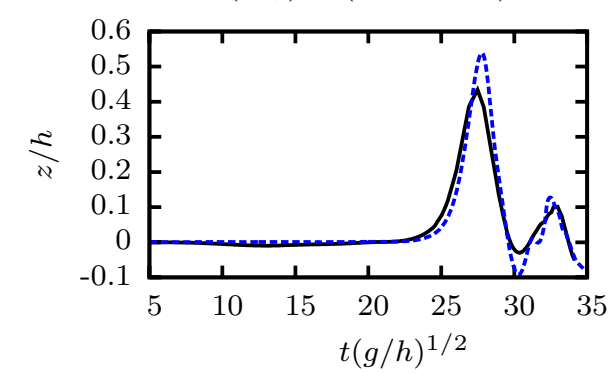

$(x, y)=(5.10,1.978)$
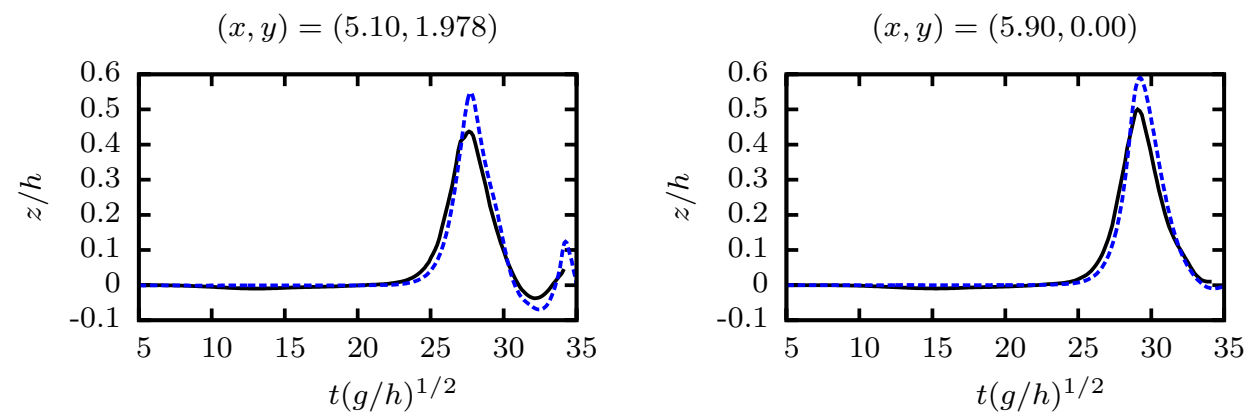

Figure 15: Time history of free surface elevation at different locations. 

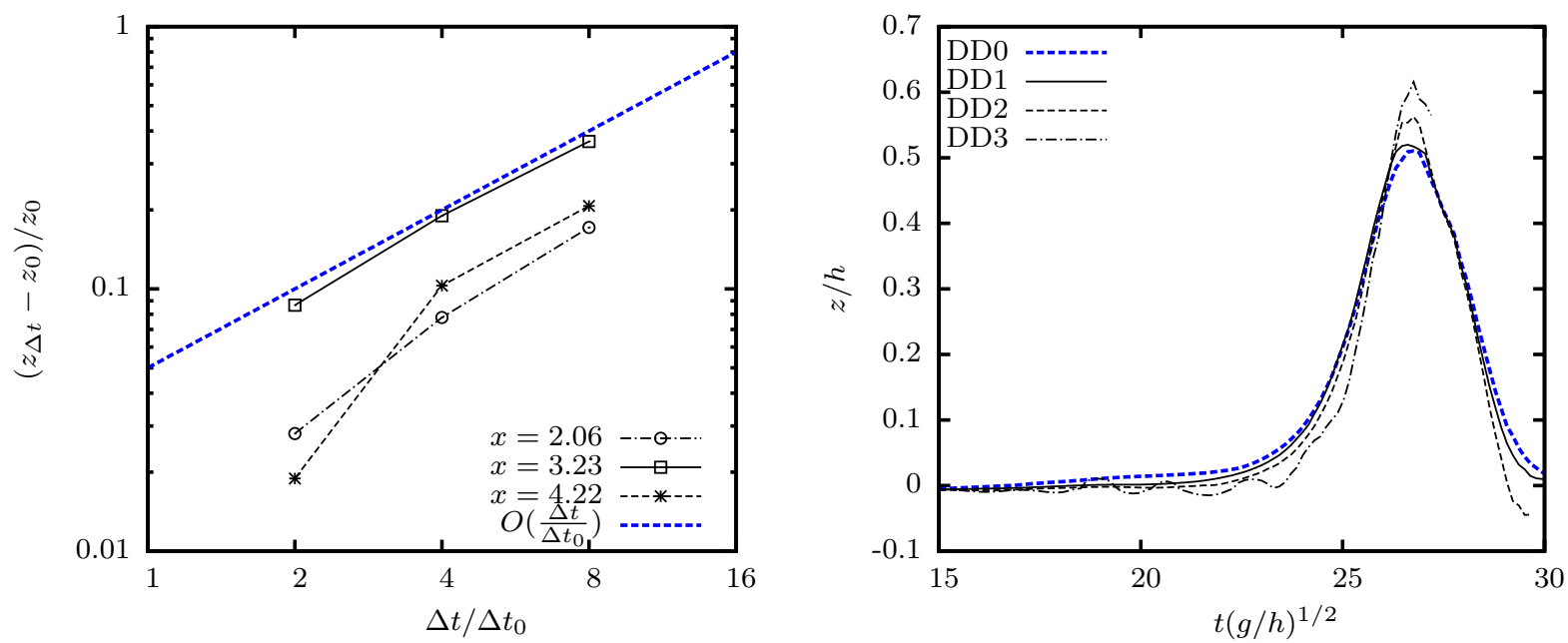

Figure 16: Effect of time stepping in wave height at different locations on the centerline plane $y=0$.. Left: convergence of maximum elevation $z_{\Delta t}$ for different time step $\Delta z$ 's, to the DD0 results, denoted as $z_{0}$, is shown to be of linear order. Right: surface elevation time history at the front of the instrumented cylinder $((x, y)=(4.22,0.00))$.

completely modeled by the NSE subdomain and computed by the FEM. Thus one can estimate the efficiency of the DD method by examining the wall time of these tests, shown in Figure 21. Notice for this example the DD method in DD0 has reduced the simulation time from DD5 for more than $50 \%$. This wall time saving is expected to be even greater when the entire domain is solved by the FEM viscous solver, in which modeling piston movement by moving mesh requires more cost. Therefore it is anticipated that significantly larger savings would result for practical large-scale problems.

\section{Conclusions}

In this study, we have introduced a 3D heterogeneous incompressible flow model that combines viscous flows and potential flows to model free surface wave impact problems. The solution scheme is based on a nonoverlapping domain decomposition (DD) method in which the coupled problem is reduced to an ODE (Bernoulli's equation) at the interface. Due to the nonlinear and transient nature of the target problem, we proposed an explicit method for the time integration of this ODE. This gives a staggered scheme for the coupled problem and avoids in-time iterations. The subdomain problems of the potential flow and the viscous flow are solved using a boundary element method (BEM) and a finte element method (FEM), respectively. The data transfer across the nonconforming mesh (quadrilateral structured mesh for BEM and triangle unstructured mesh for FEM) is done by using the same interpolation space of each subdomain. The reconstruction of the free surface profile near the interface by interpolation is also analyzed for the error of the wave height using solitary waves. We have also provided an interpretation of the matching conditions and the corresponding operators using the Helmholtz decomposition. In both numerical examples, the results of the computation wall-time have shown a significant improvement of the efficiency of the coupled scheme against the simulations based on solving the entire domain using the viscous flow model.

As shown by complexity analysis and numerical examples, the DD method is more efficient than modeling the whole flow domain using viscous flow. At the same time, the capability of the viscous flow solver in predicting impact flow pattern and force is well maintained, as shown in two examples. The pressure and drag force, both at the impact and the following quasi-static stage, are accurately predicted. The free surface profile is also accurately maintained in the whole domain.

To our knowledge this is the first implementation that combines the wave generation of a potential flow solver and viscous flow features of a Navier-Stokes Equations (NSE) solver, with both solvers equipped with free surface tracking (BEM)/capturing (FEM) capability, in building a 3D numerical wave tank (NWT). The 

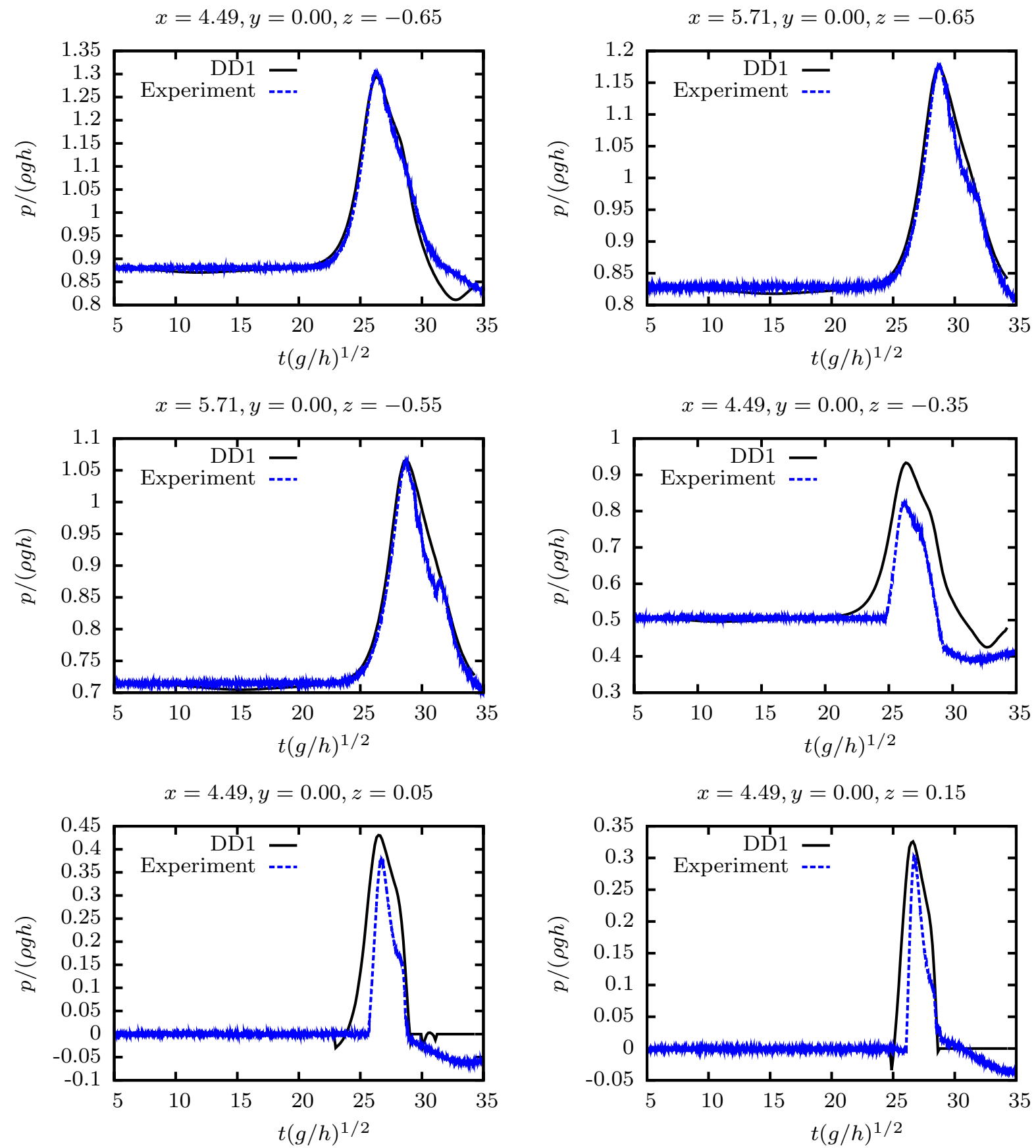

Figure 17: Time history of impact pressure on the instrumented cylinder. 

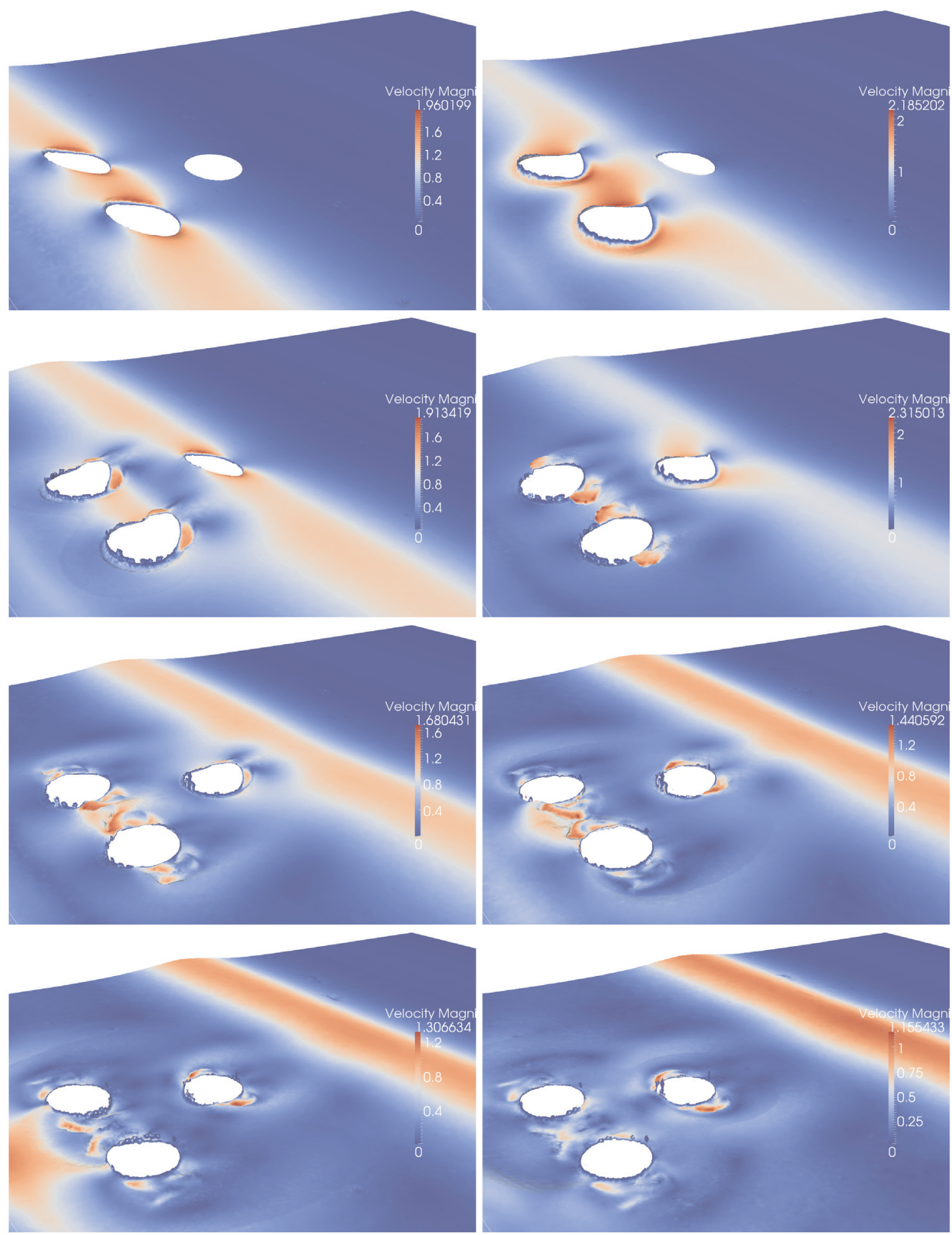

Figure 18: Free surface velocity magnitude during impact. From left-top to right-bottom: $t(g / h)^{1 / 2}=24.6,26.0,27.5,28.9$, $30.4,31.8,33.3$ and 34.7 . 


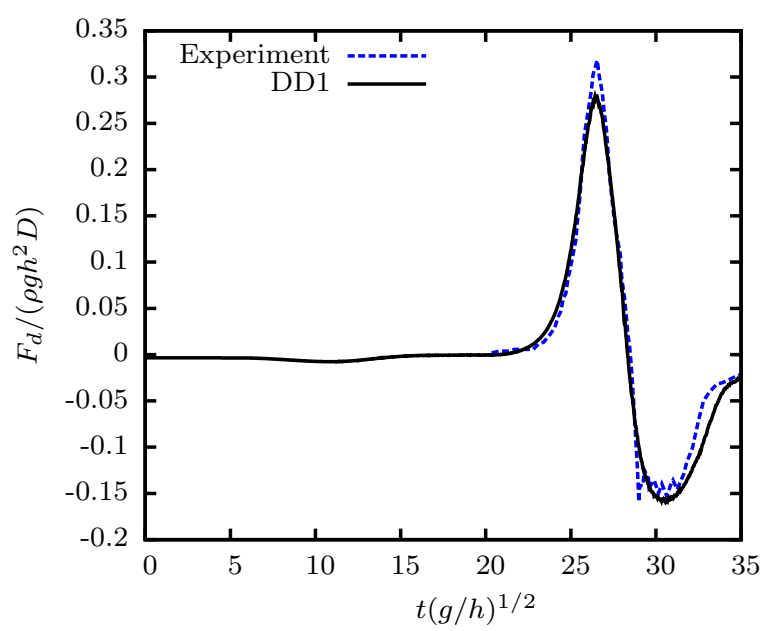

Figure 19: Time history of drag force on the instrumented cylinder.
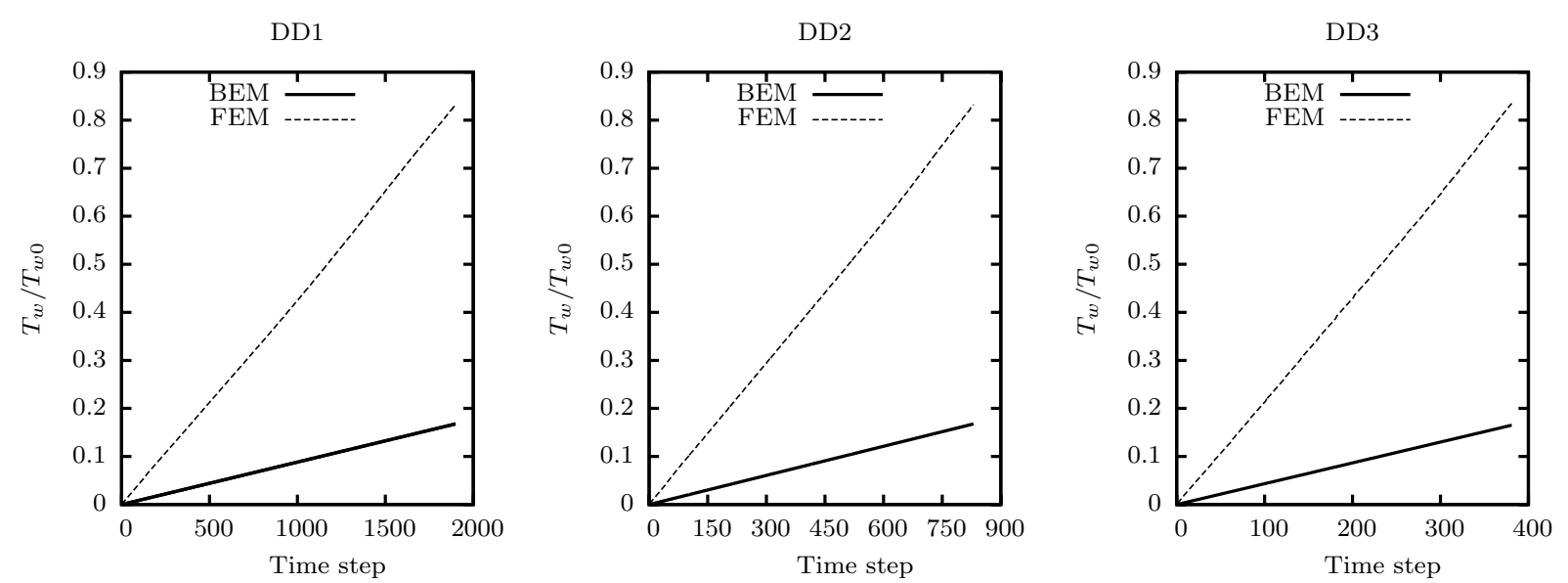

Figure 20: Accumulated wall time for different time step setting in solitary wave impacting cylinders. 


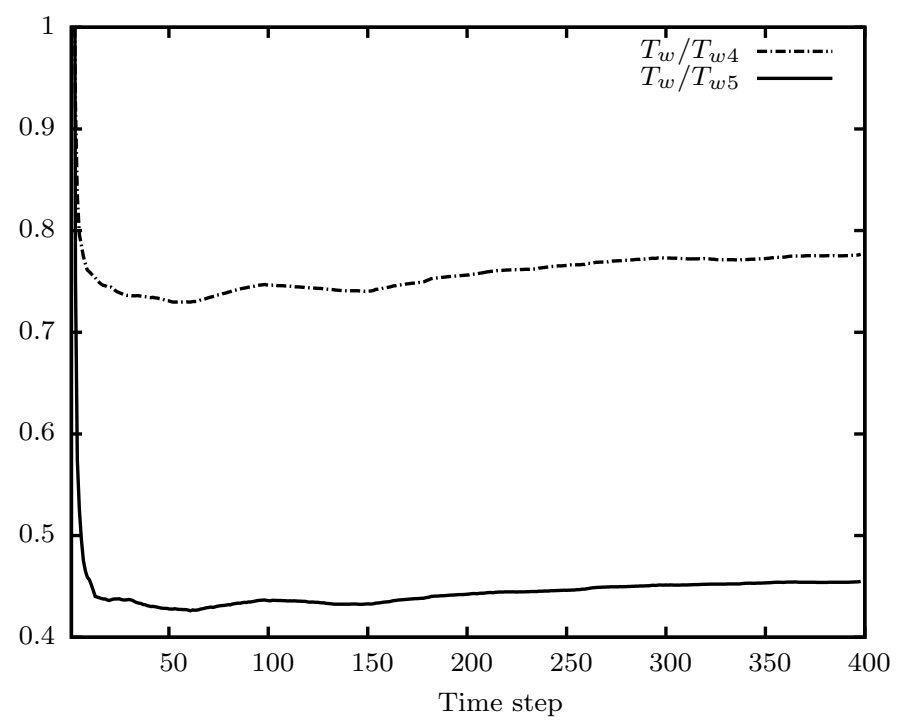

Figure 21: Ratio between accumulated wall time. $T_{w}, T_{w 4}$ and $T_{w 5}$ are the wall time for case DD0, DD4 and DD5, respectively. The ratio tends toward a constant after initial latency (such as $\mathrm{I} \backslash \mathrm{O}$ ).

DD framework gives us many options for improvement, such as higher-order implicit time integration , more accurate interface wave profile reconstruction, and other means of data transfer across the interface (e.g., mortar-based method). These improvements could extend this DD modeling environment from the current relatively short-time to long-duration simulations, thus becoming an efficient tool for other practical free surface fluid-structure interaction applications.

\section{Acknowledgement}

The first and the second authors are thankful for the Office of Naval Research ONR-N00014-11-1-0094 and N00014-13-1-0849, and the Department of Energy DE-FG36-08GO18179 for the financial support that made this work possible.

\section{Appendix A. FEM for the Navier-Stokes Equations}

Here we consider numerical approximation to the weak solutions to (1a)-(1b) with velocity boundary condition (1c), using the viscous incompressible flow Finite Element Method (FEM) solver based on the orthogonal sub-scales method (OSS) [50, 51].

Let $L^{2}\left(\Omega_{1}\right)$ denote the space of square integrable functions on $\Omega_{1}$. Next let $H^{1}\left(\Omega_{1}\right)$ be the standard Sobolev space of $L^{2}\left(\Omega_{1}\right)$ functions with gradients in $L^{2}\left(\Omega_{1}\right)$ [69], and $H_{0}^{1}\left(\Omega_{1}\right)$ its subspace of functions with compact support on $\Omega_{1}$. Let their vector counterparts be $\boldsymbol{H}^{1}(\cdot)=\left(H^{1}(\cdot)\right)^{3}$.

The domain $\Omega_{1}$ is discretized with a conforming finite element grid. We consider the finite element spaces $\boldsymbol{V}_{h} \subset \boldsymbol{H}^{1}\left(\Omega_{1}\right), Q_{h} \subset H^{1}\left(\Omega_{1}\right), \boldsymbol{V}_{h, 0} \subset \boldsymbol{H}_{0}^{1}\left(\Omega_{1}\right)$, which contain piecewise linear polynomials associated with the triangulation of $\Omega_{1}$, i.e., as usually denoted, $P 1-P 1$ finite elements. The unknowns in $\boldsymbol{V}_{h}$ have degress of freedom in the closure of $\Omega_{1}$, and those in $\boldsymbol{V}_{h, 0}$ have degrees of freedom only in the interior of $\Omega_{1}$.

At every time step $t^{n+1}$ we seek the numerical approximation $\left(\boldsymbol{u}_{h}^{n+1}, p_{h}^{n+1}\right) \in \boldsymbol{V}_{h} \times Q_{h}$ to the weak solutions $\left(\boldsymbol{u}^{n+1}, p^{n+1}\right) \in \boldsymbol{V}_{h} \times Q_{h}$. The equations to be solved for $\left(\boldsymbol{u}_{h}^{n+1}, p_{h}^{n+1}\right)$ follow as in [46, 70] where operator-splitting and stabilization have been applied to the weak form of NSE. One also determines some auxiliary variables $\left(\boldsymbol{\pi}_{h}^{n+1}, \boldsymbol{\xi}\right) \in \boldsymbol{V}_{h, 0} \times \boldsymbol{V}_{h, 0}$ used in the stabilization, and $\tau$ is the stabilization parameter (intrinsic time). The intrinsic time $\tau$ is dictated by the stability and should be calculated element by element, following the results by Behr and Tezduyar [71] as well as Franca and Frey [72]. Since the system to be 
solved for $\left(\boldsymbol{u}_{h}^{n+1}, p_{h}^{n+1}\right)$ is nonlinear and coupled, one uses Gauss-Seidel iteration which decouples the velocity and pressure equations.

We seek $\left(\boldsymbol{u}_{h}^{n+1}, p_{h}^{n+1}, \boldsymbol{\pi}_{h}^{n+1}, \boldsymbol{\xi}_{h}^{n+1}\right)$ in $\boldsymbol{V}_{h} \times Q_{h} \times \boldsymbol{V}_{h, 0} \times \boldsymbol{V}_{h, 0}$ that satisfy, in each iteration $(i)$,

$$
\begin{aligned}
& \frac{1}{\Delta t}\left(\boldsymbol{u}_{h}^{n+1,(i)}-\boldsymbol{u}_{h}^{n}, \boldsymbol{v}_{h}\right)+\left(\boldsymbol{u}_{h}^{n+\vartheta,(i-1)} \cdot \nabla \boldsymbol{u}_{h}^{n+\vartheta,(i)}, \boldsymbol{v}_{h}\right) \\
& +\left(\mu \nabla \boldsymbol{u}_{h}^{n+\vartheta,(i)}, \nabla \boldsymbol{v}_{h}\right)-\left(p_{h}^{n+1,(i-1)}, \nabla \cdot \boldsymbol{v}_{h}\right) \\
& +\left(\tau\left(\boldsymbol{u}_{h}^{n+\vartheta,(i-1)} \cdot \nabla \boldsymbol{u}_{h}^{n+\vartheta,(i)}-\boldsymbol{\pi}_{h}^{n+\vartheta,(i-1)}\right), \boldsymbol{u}_{h}^{n+\vartheta,(i-1)} \cdot \nabla \boldsymbol{v}_{h}\right) \\
& =\left(\boldsymbol{f}^{n+\vartheta}, \boldsymbol{v}_{h}\right), \forall \boldsymbol{v}_{h} \in \boldsymbol{V}_{h}, \\
& \Delta t\left(\nabla p_{h}^{n+1,(i)}-\nabla p_{h}^{n+1,(i-1)}, \nabla q_{h}\right)+\left(\tau\left(\nabla p_{h}^{n+1,(i)}-\boldsymbol{\xi}^{n+1,(i-1)}\right), \nabla q_{h}\right) \\
& =-\left(\nabla \cdot \boldsymbol{u}_{h}^{n+1,(i)}, q_{h}\right), \forall q_{h} \in Q_{h}, \\
& \left(\boldsymbol{\pi}_{h}^{n+\vartheta,(i)}, \tilde{\boldsymbol{v}}_{h}\right)=\left(\boldsymbol{u}_{h}^{n+\vartheta,(i)} \cdot \nabla \boldsymbol{u}_{h}^{n+\vartheta,(i)}, \tilde{\boldsymbol{v}}_{h}\right), \forall \tilde{\boldsymbol{v}}_{h} \in \boldsymbol{V}_{h, 0}, \\
& \left(\boldsymbol{\xi}^{n+1,(i)}, \tilde{\boldsymbol{v}}_{h}\right)=\left(\nabla p_{h}^{n+1,(i)}, \tilde{\boldsymbol{v}}_{h}\right), \forall \tilde{\boldsymbol{v}}_{h} \in \boldsymbol{V}_{h, 0} .
\end{aligned}
$$

Here $(f, g) \triangleq \int_{\Omega_{1}} f g$ is the standard $L^{2}$-inner product or its vector counterpart wherever appropriate. The scheme treats the advection term in an implicit manner, thus it allows large time steps. The system is solved for $i=1,2, \ldots$, with the data at $i=0$ given from previous time step. Typically we use $\vartheta=1 / 2$.

The unknowns $\left(\boldsymbol{u}_{h}^{n+1}, p_{h}^{n+1}\right)$ are determined by their degrees of freedom, i.e., the nodal values at the nodes of the triangulation covering $\Omega_{1}$, and we denote these by the vectors $U^{n+1}, P^{n+1}$, respectively. The algebraic form of equation (Appendix A.1)) comes from identifying the standard finite element matrices and rewriting that system for $U^{n+1}, P^{n+1}$ only, thereby eliminating the stabilization variables given from (Appendix A.1c)-(Appendix A.1d). We recall the usual notation for the finte element mass matrix and refer for the remaining details and analysis to [46, 48-51].

Let $M$ be the standard mass matrix, i.e., $M_{r q} \triangleq\left(\xi_{r}, \xi_{q}\right)$ where $\xi_{r}, \xi_{q}$ are two of the basis functions that span $\boldsymbol{V}_{h}$, and $r, q$ are the indices of some nodes of the FE grid. Thus the first term in (Appendix A.1a) can be written as $\frac{1}{\Delta t} M\left(U^{n+1}-U^{n}\right)$. Similarly, we let $K$ be the matrix associated with the advective and viscous terms, $B$ the matrix associated with the pressure gradient, $E$ the vector for the right hand side (RHS) of (Appendix A.1a), $L$ the standard discrete Laplacian matrix and $D$ the divergence matrix $\left(D=B^{T}\right)$. Next, the matrices $\tilde{D}, \tilde{M}$ and $\tilde{B}$ are the counterparts of $D, M$ and $B$ calculated over $\boldsymbol{V}_{h, 0}$ instead of $\boldsymbol{V}_{h}$.

The system to be solved for the degrees of freedom $U, P$ and equivalent to the part of (Appendix A.1) defining these variables is

$$
\begin{aligned}
& \frac{1}{\Delta t} M\left(U^{n+1}-U^{n}\right)+K\left(U^{n+\vartheta}\right) U^{n+\vartheta}-B P^{n+1}=E^{n+\vartheta} \\
& D U^{n+1}+\tau\left(L-\tilde{D} \tilde{M}^{-1} \tilde{B}\right) P^{n+1}=0
\end{aligned}
$$

where we have dropped the index of the iteration and have assumed it had converged. Note that the term $K\left(U^{n+\vartheta}\right) U^{n+\vartheta}$ associated with $K$ is nonlinear, i.e., $K$ depends on $U^{n+\vartheta}$, as the notation indicates.

We can write (Appendix A.2) as the following generalized saddle point problem

$$
\left[\begin{array}{cc}
F\left(U^{n+1}\right) & B \\
D & -C
\end{array}\right]\left[\begin{array}{l}
U^{n+1} \\
P^{n+1}
\end{array}\right]=\left[\begin{array}{l}
f \\
0
\end{array}\right]
$$

where $F=F\left(U^{n+1}\right)$ contains the contributions of $M$ and $K\left(U^{n+\vartheta}\right)$, and $C=-\tau\left(L-\tilde{D} \tilde{M}^{-1} \tilde{B}\right)$ is the stabilization matrix. The vector $f$ on the right-hand side of (Appendix A.3) contains the contribution of given data. 


\section{Appendix B. BEM for potential flow}

The PF problem is solved using a BEM proposed by Grilli et al. [10, 44], Fochesato and Dias [45] and Nimmala et al. [13]. The BEM is based on the boundary integral equation (BIE) of the Laplace's equation, see, e.g., [73]). The BIE to be solved on $\partial \Omega_{2}$ is

$$
\begin{aligned}
\alpha(\boldsymbol{x}) \varphi(\boldsymbol{x}) & =\int_{\partial \Omega_{2}} \frac{\partial \varphi(\overline{\boldsymbol{x}})}{\partial \boldsymbol{n}} G_{\boldsymbol{x}}(\overline{\boldsymbol{x}})-\varphi(\overline{\boldsymbol{x}}) \frac{\partial G_{\boldsymbol{x}}(\overline{\boldsymbol{x}})}{\partial \boldsymbol{n}} d \Gamma \\
& =\int_{\Gamma_{2}^{D}} \frac{\partial \varphi(\overline{\boldsymbol{x}})}{\partial \boldsymbol{n}} G_{\boldsymbol{x}}(\overline{\boldsymbol{x}})-\varphi^{*}(\overline{\boldsymbol{x}}) \frac{\partial G_{\boldsymbol{x}}(\overline{\boldsymbol{x}})}{\partial \boldsymbol{n}} d \Gamma \int_{\Gamma_{2}^{N}} g^{*}(\overline{\boldsymbol{x}}) G_{\boldsymbol{x}}(\overline{\boldsymbol{x}})-\varphi(\overline{\boldsymbol{x}}) \frac{\partial G_{\boldsymbol{x}}(\overline{\boldsymbol{x}})}{\partial \boldsymbol{n}} d \Gamma, \quad \boldsymbol{x} \in \partial \Omega_{2} .
\end{aligned}
$$

Here $\alpha(\boldsymbol{x})=\frac{1}{2}$, and $G_{\boldsymbol{x}}(\overline{\boldsymbol{x}})$ is the Green's function for $\mathbb{R}^{2}, G_{\boldsymbol{x}}(\overline{\boldsymbol{x}})=\frac{1}{2 \pi} \ln |\overline{\boldsymbol{x}}-\boldsymbol{x}|$.

Here we use the classical collocation BEM [74]. The boundary $\partial \Omega_{2}$ is discretized into a collection of elements $\Gamma_{i}, i=1,2 \ldots, N$ which are parts of the trace of a Cartesian grid over $\Omega_{2}$. (The grid over $\partial \Omega_{2}$ is redefined each time when the new free surface information is available). The unknowns are values of the variables at the vertices of $\Gamma_{i}$, so that (Appendix B.1) is satisfied pointwise. Specifically, we decompose $\Gamma_{D}$ into elements $\Gamma_{D, j}, j=1, \ldots, N_{D}$, and $\Gamma_{N}$ into elements $\Gamma_{N, j}, j=1, \ldots, N_{N}$, so that $N=N_{D}+N_{N}$. We let $\varphi_{i} \triangleq \varphi\left(\boldsymbol{x}_{i}\right), \psi_{i} \triangleq \psi\left(\boldsymbol{x}_{i}\right) \boldsymbol{x}_{i} \in \Gamma$, and $G_{i} \triangleq G_{\boldsymbol{x}_{i}}$.

The collocation version of (Appendix B.1) is as follows

$$
\begin{aligned}
\alpha \varphi_{i} & =\sum_{j=1}^{N_{N}} \psi_{j}^{*} \int_{\Gamma_{N, j}} G_{i} d \Gamma-\sum_{j=1}^{N_{N}} \varphi_{j} \int_{\Gamma_{N, j}} \frac{\partial G_{i}}{\partial n} d \Gamma \\
& +\sum_{j=1}^{N_{D}} \psi_{j} \int_{\Gamma_{D, j}} G_{i} d \Gamma-\sum_{j=1}^{N_{D}} \varphi_{j}^{*} \int_{\Gamma_{D, j}} \frac{\partial G_{i}}{\partial n} d \Gamma, \quad i=1,2, \ldots, N .
\end{aligned}
$$

(Appendix B.2)

Collecting the nodal unknowns $\Phi \triangleq\left(\varphi_{i}\right)_{i=1}^{N_{D}}, \Psi \triangleq\left(\psi_{j}\right)_{j=1}^{N_{N}}$ we formulate the linear system that needs to be solved in BEM. We define

$$
\begin{aligned}
J_{i, j}^{N} & =\int_{\Gamma_{D, j}} G_{i} d \Gamma, \boldsymbol{x}_{i} \in \Gamma_{N} ; \quad J_{i, j}^{N^{*}}=\int_{\Gamma_{N, j}} G_{i} d \Gamma, \boldsymbol{x}_{i} \in \Gamma_{N}, \\
K_{i, j}^{N^{*}} & =\int_{\Gamma_{D, j}} \frac{\partial G_{i}}{\partial n} d \Gamma, \boldsymbol{x}_{i} \in \Gamma_{N} ; \quad J_{i, j}^{D}=\int_{\Gamma_{D, j}} G_{i} d \Gamma, \boldsymbol{x}_{i} \in \Gamma_{D}, \\
J_{i, j}^{D^{*}} & =\int_{\Gamma_{N, j}} G_{i} d \Gamma, \boldsymbol{x}_{i} \in \Gamma_{D} ; \quad K_{i, j}^{D}=\int_{\Gamma_{N, j}} \frac{\partial G_{i}}{\partial n} d \Gamma, \boldsymbol{x}_{i} \in \Gamma_{D} ; \\
K_{i, j}^{N} & =\int_{\Gamma_{N, j}} \frac{\partial G_{i}}{\partial n} d \Gamma+\alpha \delta_{i j}, \quad \boldsymbol{x}_{i} \in \Gamma_{N} ; \\
K_{i, j}^{D^{*}} & =\int_{\Gamma_{D, j}} \frac{\partial G_{i}}{\partial n} d \Gamma+\alpha \delta_{i j}, \boldsymbol{x}_{i} \in \Gamma_{D} .
\end{aligned}
$$

Then (Appendix B.2) is equivalent to

$$
\left[\begin{array}{ll}
K^{N} & -J^{N} \\
K^{D} & -J^{D}
\end{array}\right]\left[\begin{array}{l}
\Phi \\
\Psi
\end{array}\right]=\left[\begin{array}{l}
J^{N^{*}} \Psi^{*}-K^{N^{*}} \Phi^{*} \\
J^{D^{*}} \Psi^{*}-K^{D^{*}} \Phi^{*}
\end{array}\right]
$$

(Appendix B.3)

\section{References}

[1] K. Kleefsman, G. Fekken, A. Veldman, B. Iwanowski, B. Buchner, A volume-of-fluid based simulation method for wave impact problems, Journal of Computational Physics 206 (1) (2005) 363-393.

[2] B. Peseux, L. Gornet, B. Donguy, Hydrodynamic impact: Numerical and experimental investigations, Journal of Fluids and Structures 21 (3) (2005) 277-303. 
[3] S. Yim, M. Olsen, C. K.F., A. M., Tsunami modeling, fluid load simulation and validation using geospatial data, Journal of Structural Engineering, ASCE.

[4] M. S. Longuet-Higgins, E. D. Cokelet, The deformation of steep surface waves on water. i. a numerical method of computation, Proceedings of the Royal Society of London. A. Mathematical and Physical Sciences 350 (1660) (1976) 1-26.

[5] P. Lin, P. L. Liu, A numerical study of breaking waves in the surf zone, Journal of Fluid Mechanics 359 (-1) (1998) 239264

[6] J.-C. Park, M.-H. Kim, H. Miyata, Fully non-linear free-surface simulations by a 3D viscous numerical wave tank, International Journal for Numerical Methods in Fluids 29 (6) (1999) 685703.

[7] H. Yuan, C. H. Wu, An implicit three-dimensional fully non-hydrostatic model for free-surface flows, International Journal for Numerical Methods in Fluids 46 (7) (2004) 709733.

[8] T. Li, P. Troch, J. De Rouck, Wave overtopping over a sea dike, Journal of Computational Physics 198 (2) (2004) $686-726$.

[9] S. Beji, J. Battjes, Numerical simulation of nonlinear wave propagation over a bar, Coastal Engineering 23 (12) (1994) 1-16.

[10] S. Grilli, P. Guyenne, F. Dias, A fully non-linear model for three-dimensional overturning waves over an arbitrary bottom, International Journal for Numerical Methods in Fluids 35 (7) (2001) 829-867.

[11] S. Ryu, M. H. Kim, P. J. Lynett, Fully nonlinear wave-current interactions and kinematics by a BEM-based numerical wave tank, Computational Mechanics 32 (4-6) (2003) 336-346.

[12] P. Liu, G. Simarro, J. Vandever, A. Orfila, Experimental and numerical investigation of viscous effects on solitary wave propagation in a wave tank, Coastal Engineering 53 (23) (2006) 181-190.

[13] S. Nimmala, S. Yim, S. Grilli, An efficient parallelized 3d fnpf numerical wave tank for large-scale wave basin experiment simulation, Offshore Mechanics and Arctic Engineering, ASME 135.

[14] C. H. Kim, A. H. Clement, K. H. Tanizawa, Recent research and development of numerical wave tanks-a review, International Journal of Offshore and Polar Engineering 9 (4).

[15] A. M. Quarteroni, A. Valli, Domain Decomposition Methods for Partial Differential Equations, 1st Edition, Oxford University Press, 1999.

[16] D. E. Keyes, L. C. McInnes, C. Woodward, W. Gropp, E. Myra, M. Pernice, J. Bell, J. Brown, A. Clo, J. Connors, E. Constantinescu, D. Estep, K. Evans, C. Farhat, A. Hakim, G. Hammond, G. Hansen, J. Hill, T. Isaac, X. Jiao, K. Jordan, D. Kaushik, E. Kaxiras, A. Koniges, K. Lee, A. Lott, Q. Lu, J. Magerlein, R. Maxwell, M. McCourt, M. Mehl, R. Pawlowski, A. P. Randles, D. Reynolds, B. Rivire, U. Rde, T. Scheibe, J. Shadid, B. Sheehan, M. Shephard, A. Siegel, B. Smith, X. Tang, C. Wilson, B. Wohlmuth, Multiphysics simulations: Challenges and opportunities, International Journal of High Performance Computing Applications 27 (1) (2013) 4-83.

[17] Q. Chen, J. T. Kirby, R. A. Dalrymple, A. B. Kennedy, A. Chawla, Boussinesq modeling of wave transformation, breaking, and runup. II: 2D, Journal of Waterway, Port, Coastal, and Ocean Engineering 126 (1) (2000) 4856

[18] H. Lamb, Hydrodynamics, 6th Edition, Cambridge University Press, 1993.

[19] J. J. Stoker, Water Waves: The Mathematical Theory with Applications, John Wiley \& Sons, 1957.

20] P. Bjørstad, O. Widlund, Iterative methods for the solution of elliptic problems on regions partitioned into substructures, SIAM Journal on Numerical Analysis 23 (6) (1986) 1097-1120.

[21] L. Cambier, W. Ghazzi, J. P. Veuillot, H. Viviand, A multi-domain approach for the computation of viscous transonic flows by unsteady type methods, ONERA TP 66.

[22] Q. V. Dinh, R. Glowinski, J. Periaux, G. Terrasson, On the coupling of viscous and inviscid models for incompressible fluid flows via domain decomposition, in: Proceedings of the First International Symposium on Domain Decomposition Methods for Partial Differential Equations, Ecole Nationale des Ponts et Chausses, Paris, France, 1988, pp. 350-369.

[23] M. Dryja, A finite element capacitance method for elliptic problems on regions partitioned into subregions, Numerische Mathematik 44 (2) (1984) 153-168.

[24] R. Glowinski, J. Periaux, Q. V. Dinh, Domain decomposition methods for nonlinear problems in fluid dynamics, Computer Methods in Applied Mechanics and Engineering 40.

[25] J.-C. Xu, J. Zou, Some nonoverlapping domain decomposition methods, SIAM Review 40 (4) (1998) 857-914.

[26] J.-C. Xu, Iterative methods by space decomposition and subspace correction, SIAM Review 34 (4) (1992) 581-613.

[27] T. P. A. Mathew, Domain Decomposition Methods for the Numerical Solution of Partial Differential Equations, 1st Edition, Springer, 2008

[28] A. Toselli, O. B. Widlund, Domain Decomposition Methods-algorithms and Theory, Springer, 2005

[29] B. I. Wohlmuth, Discretization Methods and Iterative Solvers Based on Domain Decomposition, 1st Edition, Springer, 2001.

[30] L. Fatone, P. Gervasio, A. Quarteroni, Multimodels for incompressible flows, Journal of Mathematical Fluid Mechanics 2 (2) (2000) 126-150.

[31] L. Fatone, P. Gervasio, A. Quarteroni, Multimodels for incompressible flows: iterative solutions for the navier-Stokes/Oseen coupling, Mathematical Modelling and Numerical Analysis 35 (3) (2001) 26.

[32] M. Feistauer, C. Schwab, On coupled problems for viscous flow in exterior domains, Tech. Rep. 96-07, Eidgenössische Technische Hochschule, Zürich, Switzerland (1996).

[33] M. Feistauer, C. Schwab, Coupling of an interior NavierStokes problem with an exterior oseen problem, Tech. Rep. 98-01, Eidgenössische Technische Hochschule, Zürich Switzerland (1998).

[34] K. Schenk, F. K. Hebeker, Coupling of two dimensional viscous and inviscid incompressible stokes equations, Tech. Rep. Technical report preprint 93-68 (SFB 359), Heidelberg University (1993).

[35] E. Campana, A. Di Mascio, P. G. Esposito, F. Lalli, Viscous-inviscid coupling in free surface ship flows, International Journal for Numerical Methods in Fluids 21 (9) (1995) 699-722.

[36] A. Iafrati, E. F. Campana, A domain decomposition approach to compute wave breaking (wave-breaking flows), International Journal for Numerical Methods in Fluids 41 (4) (2003) 419-445. 
[37] G. Colicchio, M. Greco, O. M. Faltinsen, A BEM-level set domain-decomposition strategy for non-linear and fragmented interfacial flows, International Journal for Numerical Methods in Engineering 67 (10) (2006) 1385-1419.

[38] K. I. Sitanggang, P. J. Lynett, Multi-scale simulation with a hybrid boussinesq-RANS hydrodynamic model, International Journal for Numerical Methods in Fluids 62 (9) (2010) 1013-1046.

[39] S. Kim, M. Yamashiro, A. Yoshida, A simple two-way coupling method of BEM and VOF model for random wave calculations, Coastal Engineering 57 (1112) (2010) 1018-1028.

[40] J. A. Hamilton, R. W. Yeung, Viscous and inviscid matching of three-dimensional free-surface flows utilizing shell functions, Journal of Engineering Mathematics 70 (1-3) (2011) 43-66.

[41] Y. Zhang, F. Del-Pin, S. Yim, A heterogeneous flow model based on domain decomposition method for free-surface fluid-structure interaction problems, International Journal for Numerical Methods in Fluids.

[42] Y. Zhang, M. Peszynska, S. Yim, Coupling of Viscous and Potential Flow Models with Free Surface for Near and Far Field Wave Propagation, International Journal of Numerical Analysis and Modeling, Series B 4 (3) (2013) $256-282$.

[43] J. H. Bramble, J. E. Pasciak, A. H. Schatz, An iterative method for elliptic problems on regions partitioned into substructures, Mathematics of Computation 46 (174) (1986) 361-369.

[44] S. T. Grilli, R. Subramanya, Numerical modeling of wave breaking induced by fixed or moving boundaries, Computational Mechanics 17 (6) (1996) 374-391.

[45] C. Fochesato, F. Dias, A fast method for nonlinear three-dimensional free-surface waves, Proceedings of the Royal Society A: Mathematical, Physical and Engineering Science 462 (2073) (2006) 2715.

[46] O. Soto, R. Löhner, J. Cebral, An implicit monolithic time accurate finite element scheme for incompressible flow problems, in: Proceedings of the AIAA 15th Computational Fluid Dynamics Conference, AIAA 2001-2616, AIAA, Anaheim, California, 2001, pp. 1-11.

[47] O. Soto, R. Löhner, F. Camelli, A linelet preconditioner for incompressible flow solvers, International Journal of Numerical Methods for Heat \& Fluid Flow 13 (1) (2003) 133-147.

[48] O. Soto, R. Löhner, J. Cebral, F. Camelli, A stabilized edge-based implicit incompressible flow formulation, Computer Methods in Applied Mechanics and Engineering 193 (23-26) (2004) 2139-2154.

[49] R. Codina, Pressure stability in fractional step finite element methods for incompressible flows, Journal of Computational Physics 170 (1) (2001) 112-140.

[50] R. Codina, Stabilization of incompressibility and convection through orthogonal sub-scales in finite element methods, Computer Methods in Applied Mechanics and Engineering 190 (13-14) (2000) 1579-1599.

[51] R. Codina, Stabilized finite element approximation of transient incompressible flows using orthogonal subscales, Computer Methods in Applied Mechanics and Engineering 191 (39-40) (2002) 4295-4321.

[52] O. A. Ladyzhenskaya, The mathematical theory of viscous incompressible flow, enlarged 2nd Edition, Gordon and Breach, 1969.

[53] I. Babuška, The finite element method with lagrangian multipliers, Numerische Mathematik 20 (3) (1973) $179-192$.

[54] F. Brezzi, On the existence, uniqueness and approximation of saddle-point problems arising from lagrangian multipliers, RAIRO Anal. Numer 8 (2) (1974) 129151.

[55] R. Dean, R. Dalrymple, Water wave mechanics for engineers and scientists, Vol. 2nd of Advanced Series on Ocean Engineering, World Scientific, 1991.

[56] S. A. Hughes, Physical Models and Laboratory Techniques in Coastal Engineering, World Scientific, 1993.

[57] B. N. Khoromskij, G. Wittum, B. N. Khoromskij, Numerical Solution of Elliptic Differential Equations by Reduction to the Interface, 1st Edition, Springer, 2004.

[58] M. Dryja, O. Widlund, Towards a unified theory of domain decomposition algorithms for elliptic problems, in: Proceedings of the third international symposium on Domain Decomposition Methods for Partial Differential Equations, SIAM, Houston, Texas, 1989, pp. 3-21.

[59] S. R. Wu, L. Gu, Introduction to the explicit finite element method for nonlinear transient dynamics, Wiley, Hoboken, N.J., 2012.

[60] P. M. Gresho, R. L. Sani, On pressure boundary conditions for the incompressible navier-stokes equations, International Journal for Numerical Methods in Fluids 7 (10) (1987) 1111-1145.

[61] P. M. Gresho, Incompressible fluid dynamics: some fundamental formulation issues, Annual Review of Fluid Mechanics 23 (1) (1991) 413453.

[62] D. Enright, R. Fedkiw, J. Ferziger, I. Mitchell, A hybrid particle level set method for improved interface capturing, Journal of Computational Physics 183 (1) (2002) 83116.

[63] K. Atkinson, An Introduction to Numerical Analysis, 2nd Edition, Wiley, 1989.

[64] R. S. Johnson, A Modern Introduction to the Mathematical Theory of Water Waves, first edition Edition, Cambridge University Press, 1997.

[65] H. Sohr, The Navier-Stokes Equations: An Elementary Functional Analytic Approach, Springer, 2012.

[66] D. Joseph, Helmholtz decomposition coupling rotational to irrotational flow of a viscous fluid, Proceedings of the National Academy of Sciences of the United States of America 103 (39) (2006) 14272-14277.

[67] C. Geuzaine, J.-F. Remacle, Gmsh: A 3-d finite element mesh generator with built-in pre- and post-processing facilities, International Journal for Numerical Methods in Engineering 79 (11) (2009) 13091331.

[68] S. Yim, W. Zhang, A multiphysics multiscale 3d computational wave basin model for wave impact load on a cylindrical structure, Journal of Disaster Research 4 (6) (2005) 450-461.

[69] A. M. Quarteroni, Numerical Models for Differential Problems, 1st Edition, Springer, 2009.

[70] R. Rannacher, On chorin's projection method for the incompressible navier-stokes equations, in: John G. Heywood, Kyya Masuda, Reimund Rautmann, Vsevolod A. Solonnikov (Eds.), The Navier-Stokes Equations II-Theory and Numerical 
Methods, Vol. 1530 of Lecture Notes in Mathematics, Springer, 1992, pp. 167-183.

[71] M. Behr, T. E. Tezduyar, Finite element solution strategies for large-scale flow simulations, Computer Methods in Applied Mechanics and Engineering 112 (1-4) (1994) 324.

[72] L. P. Franca, S. L. Frey, Stabilized finite element methods: II. the incompressible navier-stokes equations, Computer Methods in Applied Mechanics and Engineering 99 (2-3) (1992) 209-233.

[73] K. Atkinson, The Numerical Solution of Integral Equations of the Second Kind, Cambridge University Press, 1997.

[74] C. A. Brebbia, J. Dominguez, Boundary Elements: An Introductory Course, 2nd Edition, Computational Mechanics, 1992. 